\title{
EXTENDING REAL VALUATIONS TO SKEW POLYNOMIAL RINGS
}

\author{
A. GRANJA, M.C. MARTÍNEZ, AND C. RODRÍGUEZ
}

\begin{abstract}
Let $D$ be a division ring, $T$ be a variable over $D, \sigma$ be an endomorphism of $D, \delta$ be a $\sigma$-derivation on $D$ and $R=D[T ; \sigma, \delta]$ the left skew polynomial ring over $D$. We show that the set $\left(\operatorname{Val}_{\nu}(R), \preceq\right)$ of $\sigma$-compatible real valuations which extend to $R$ a fixed proper real valuation $\nu$ on $D$ has a natural structure of parameterized complete non-metric tree, where $\preceq$ is the partial order given by $\mu \preceq \widetilde{\mu}$ if and only if $\mu(f) \leq \widetilde{\mu}(f)$ for all $f \in R$ and $\mu, \widetilde{\mu} \in \operatorname{Val}_{\nu}(R)$.
\end{abstract}

\section{INTRODUCTION}

Throughout this paper, we will denote by $D$ a division ring, by $T$ a variable over $D$, by $\sigma$ an endomorphism of $D$, by $\delta$ a left $\sigma$-derivation (i.e. $\delta(a+b)=\delta(a)+\delta(b)$ and $\delta(a b)=$ $\sigma(a) \delta(b)+\delta(a) b$ for all $a, b \in D)$ and by $R=D[T ; \sigma, \delta]=\left\{f(T) ; f(T)=a_{n} T^{n}+\cdots+a_{0}\right\}$ the left skew polynomial ring over $D$ such that $T a=\sigma(a) T+\delta(a)$. (See chapter 2 of $[\mathrm{GW}]$ for details on skew polynomials rings.)

The aim of this paper is to study real valuations on skew polynomials rings. Namely, let $\operatorname{Val}(R)$ be the set of functions $\mu: R \rightarrow \overline{\mathbb{R}}=\mathbb{R} \cup\{\infty\}$ satisfying the standard axioms of valuations, whose restriction to $D$ is no trivial and which are $\sigma$-compatible (i.e. $\mu(\sigma(a))=\mu(a)$ for each $a \in D$ ). We consider the partial order $\preceq$ on $\operatorname{Val}(R)$ given by $\mu \preceq \widetilde{\mu}$ if and only if $\mu(f) \leq \widetilde{\mu}(f)$ for all $f \in R$ and $\mu, \widetilde{\mu} \in \operatorname{Val}(R)$. Since $\mu \preceq \widetilde{\mu}$ implies that $\mu$ and $\widetilde{\mu}$ have the same restriction to $D$ (see Remark 2.1 below), then $\operatorname{Val}(R)$ is a disjoint union of the sets $\operatorname{Val}_{\nu}(R)$ such that $\nu$ is a real valuation on $D$.

Our main result (Theorem 5.3) states that $\left(\operatorname{Val}_{\nu}(R), \preceq\right)$ is a parameterized complete non-metric tree. This was first showed by Berkovich in [Ber] (see also [BR]) under the assumptions that $D$ is an algebraically closed commutative field which is complete with respect to the $\nu$-topology and $R=D[T]$ is the polynomial ring over $D$ (i.e. $\sigma=1_{D}$ and $\delta=0$ ). Similar results are given in $[\mathrm{FJ}]$ when $D=\mathbb{C}((X))$ is the Laurent series ring over the complex field, $R=\mathbb{C}((X))[T]$ and $\nu$ is the usual order in $X$; and in [Gra2] for normalized real valuations on a two-dimensional local noetherian regular ring.

Recently, real trees as $\left(\operatorname{Val}_{\nu}(R), \preceq\right)$ has been used to introduce a Laplacian operator and study harmonic and subharmonic functions as well as dynamics of rational functions in an analogous way as in $\mathbb{C}$. (See $[\mathrm{BR}]$ and the references given there.)

On the other hand, our results can be appropriate to study rings that are particular cases of (iterated) skew polynomials rings. For example, Weyl algebras, quantum spaces, some subalgebras of Lie algebras, .... (See $[\mathrm{GW}]$ for details.) In particular, our techniques can be used to compute extensions of valuations to non commutative algebras, which are studied, for example,

Date: September 25, 2012

2000 Mathematics Subject Classification. 13A18, 13F30, 54F50, 12D05.

Key words and phrases. Valuation, parameterized tree, real rank, factorization. 
on $[\mathrm{L}]$ from other point of view. Furthermore, we think that the results of this paper can also be useful to give irreducibility criterions for skew polynomials in a similar setting out as for polynomial rings over commutative fields equipped with a proper real valuation. (See for example, $[\mathrm{CZ}],[\mathrm{GMR}]$ and $[\mathrm{HOS}]$.

It is worth pointing out that techniques of this paper are similar to MacLane one's in [M]. However, we shall put our attention on the partial order $\preceq$ rather than generalize MacLane's concept of key polynomial to skew polynomial rings, which we shall do at the end of the paper. Let us explain briefly our viewpoint.

Let $\mu, \widetilde{\mu} \in \operatorname{Val}(R)$ be such that $\mu \prec \widetilde{\mu}$ and let $\phi \in R$ be such that $\mu(\phi)<\widetilde{\mu}(\phi)$ and $\operatorname{deg}(\phi) \leq$ $\operatorname{deg}\left(\phi^{\prime}\right)$ for all $\phi^{\prime} \in R$ with $\mu\left(\phi^{\prime}\right)<\widetilde{\mu}\left(\phi^{\prime}\right)$. Here, $\operatorname{deg}(f)$ denotes the usual degree of $f \in R$. Since $\mu^{\prime}(a f)=\mu^{\prime}(f a)$ for each $\mu^{\prime} \in \operatorname{Val}(R)$ and $a, f \in R$, then $\mathcal{I}(\sigma, \delta, \mu, \widetilde{\mu}, \phi)=\min \{\mu(r(\phi, a))-$ $\mu(a) ; a \in R, 0 \leq \operatorname{deg}(a)<\operatorname{deg}(\phi)\} \geq \widetilde{\mu}(\phi)>\mu(\phi)$, where $\phi a=q(\phi, a) \phi+r(\phi, a)$ with $\operatorname{deg}(r(\phi, a))<\operatorname{deg}(\phi)$, i.e the left division of $\phi a$ by $\phi$, see [J]. (Note that $\mu(r(\phi, a))=\widetilde{\mu}(r(\phi, a))$ and $\widetilde{\mu}(a)=\mu(a)$, since $\operatorname{deg}(r(\phi, a))<\operatorname{deg}(\phi)$ and $\operatorname{deg}(a)<\operatorname{deg}(\phi)$.) We call $\mathcal{I}(\sigma, \delta, \mu, \widetilde{\mu}, \phi)$ the compatibility index of $\phi$ with respect to $\mu$ and $\widetilde{\mu}$ and we point out that $\mathcal{I}(\sigma, \delta, \mu, \widetilde{\mu}, \phi)=\infty$ when $\sigma=1_{D}$ is the identity on $D$ and $\delta=0$. This is the main difference between consider skew polynomial rings or polynomial rings over a commutative field. The above allows us to define augmented and limit valuations in a similar way as MacLane does (see section 3 bellow).

Next, we introduce the Apéry base for a valuation $\mu \in \operatorname{Val}(R)$ which is the natural generalization of the usual Apéry base for the values semigroup of an irreducible algebroid plane singularity (see for example [Gra1]). The properties of the Apéry base of $\mu$ are related to a second invariant, the iterated sequence of valuations associated with $\mu$, which describes the valuations $\mu^{\prime} \in \operatorname{Val}(R)$ such that $\mu^{\prime} \preceq \mu$. We show that the numerical part of the iterated sequence of valuations and the Apéry base are equivalent data of the valuation. With this background we get Theorem 5.3 and finally we define and study left key skew polynomials.

The paper is organized in six sections including this introduction. In the next section we review some concepts about valuations and show some properties of the partial order $\preceq$. The third section is devoted to introduce and study augmented and limit valuations in a similar way as $[\mathrm{M}]$ and [V], without considering key polynomials. Section 4 discuses the concepts of Apéry base and iterated sequence of valuations and establishes the relation between both invariants in Theorem 4.4 and Corollary 4.5. As we had said, section 5 is devoted to obtain our main result, Theorem 5.3. The last section is devoted to introduce and study the concept of left key skew polynomial. The main difference with MacLane one's is that left key skew polynomials satisfy an additional statement: the compatibility condition, which is also defined in terms of the left compatibility index. The relation between our initial setting out and left key skew polynomials is stated in Theorem 6.4. We finish the paper with two examples of left key skew polynomials with infinite left compatibility index.

\section{Ordering VALUATIONS}

In this section, we review some concepts about valuations on rings and we introduce some notation.

A valuation on $R=D[T ; \sigma, \delta]$ is a map $\mu: R \longrightarrow \overline{\mathbb{R}}$ such that

(V1) $\mu(x y)=\mu(x)+\mu(y)$ for all $x, y \in R$.

(V2) $\mu(x+y) \geq \min \{\mu(x), \mu(y)\}$ for all $x, y \in R$. 
(V3) $\mu(1)=0$ and $\mu(0)=\infty$.

Where $\overline{\mathbb{R}}=\mathbb{R} \cup\{\infty\}$ is the extended monoid of $\mathbb{R}$ by a symbol $\infty$ satisfying the usual rules $\infty+x=x+\infty=\infty$ for all $x \in \overline{\mathbb{R}}$; and $x<\infty$ for all $x \in \mathbb{R}$

If $\nu(R)=\{0, \infty\}, \nu$ is said to be trivial, otherwise $\nu$ is called no trivial or proper. Moreover, the two-side ideal $\nu^{-1}(\infty)$ of $R$ is called the support of $\nu$ and valuations with zero support are called Krull valuations.

From now on we shall make the assumption that every valuation $\mu$ on $R$ is $\sigma$-compatible (i.e. $\mu(\sigma(a))=\mu(a)$ for all $a \in D$ ) and also every real valuation on $D$ will be assumed $\sigma$ compatible.

Finally, we denote by $\operatorname{deg}(f)$ the usual degree of $f \in R$ (here $\operatorname{deg}(0)=-\infty$ ) and we also recall that if $f, g \in R$, there exist $q, r \in R$ such that $\operatorname{deg}(r)<\operatorname{deg}(g)$ and $f=q g+r$, i.e. we have a left division algorithm on $R$ (see $[\mathrm{J}]$ ).

The rest of the section is devoted to introduce and study a natural partial order $\preceq$ on the set of real valuations on $R$. Namely, let $\mu, \widetilde{\mu}: R \longrightarrow \overline{\mathbb{R}}$ be two real valuations on $R$. We write $\mu \preceq \widetilde{\mu}$ if and only if $\mu(f) \leq \widetilde{\mu}(f)$ for all $f \in R$.

Remark 2.1. Note that if $\mu \preceq \widetilde{\mu}$, then $\mu(a)=\widetilde{\mu}(a)$ for all $a \in D$ (i.e. $\mu$ and $\widetilde{\mu}$ are extensions to $R$ of the same Krull valuation $\nu$ on $D$ ). In particular, $\mu$ is trivial on $D$ if and only if $\widetilde{\mu}$ is also trivial on $D$.

Next, we shall describe the real valuations $\mu$ on $R$ whose restriction to $D$ is trivial. We have the following possibilities:

A) There exists $h \in R$ such that $\mu(h)<0$. Then $\mu(T)<0$ (note that if $\mu(T) \geq 0$, then $\mu(h) \geq 0$ for all $h \in R)$. Therefore, $\mu(h)=\operatorname{deg}(h) \mu(T)$ and $\mu$ is equivalent to the discrete valuation - deg.

B) $\mu(h) \geq 0$ for all $h \in R$. Let $\mathcal{A}_{\mu}$ be the two-side ideal of $R$ given by $\mathcal{A}_{\mu}=\{h \in R ; \mu(h)>$ $0\}$. Since $R$ is a left principal ideal domain (see $[\mathrm{J}]$ ), then $\mathcal{A}_{\mu}=R f$ for some irreducible element $f \in R$.

B1) If $\mathcal{A}_{\mu}=(0)$ then $\mu$ is a trivial valuation on $R$.

B2) If $\mathcal{A}_{\mu} \neq(0)$, then $f \neq 0$ and for all $g \in R-\{0\}$ we can write $g=h f^{n}$ with $h \notin \mathcal{A}_{\mu}$. So, $\mu(g)=\mu(h)+n \mu(f)=n \mu(f)$.

Moreover, we have the following two possibilities:

B2i) $\mu(f)<\infty$, and $\mu$ is a Krull no trivial valuation of $R$.

B2ii) $\mu(f)=\infty$, and $\mu$ is a trivial no Krull valuation.

Thus, if $\widetilde{\mu}$ is a real valuation on $R$ such that $\mu \preceq \widetilde{\mu}$ and $\mu$ is trivial on $D$, then $\widetilde{\mu}$ is trivial on $D$ by Remark 2.1 and we have three possibilities:

I) $\mu$ is of type A). In this case, $\widetilde{\mu}$ can be either of type A) or B).

II) $\mu$ is of type B1). In this case, $\widetilde{\mu}$ can be either of type B1) or B2).

III) $\mu$ is of type B2). In this case, either $\widetilde{\mu}$ is of type B2i) such that $\mu(f)<\widetilde{\mu}(f)<\infty$ or $\widetilde{\mu}$ is of type B2ii) such that $\mu(f) \leq \widetilde{\mu}(f)=\infty$. 
For the rest of the paper, we will consider real valuations on $R$ whose restriction to $D$ is a proper valuation. We will denote by $\operatorname{Val}(R)$ the set of these valuations.

We next set some notation that we shall use throughout the paper and which is similar to some one of $[\mathrm{V}]$.

Let $\mu, \widetilde{\mu} \in \operatorname{Val}(R)$ be such that $\mu \preceq \widetilde{\mu}$. We denote by $\widetilde{\Phi}(\mu, \widetilde{\mu})=\{\phi \in R ; \mu(\phi)<\widetilde{\mu}(\phi)\}$. Note that $\widetilde{\Phi}(\mu, \widetilde{\mu})=\emptyset$ if and only if $\mu=\widetilde{\mu}$. Furthermore, if $\widetilde{\Phi}(\mu, \widetilde{\mu}) \neq \emptyset$, we write:

- $d(\mu, \widetilde{\mu})=\min \{\operatorname{deg}(\phi) ; \phi \in \widetilde{\Phi}(\mu, \widetilde{\mu})\}$.

- $\Phi(\mu, \widetilde{\mu})=\{\phi \in \widetilde{\Phi}(\mu, \widetilde{\mu}) ; \operatorname{deg}(\phi)=d(\mu, \widetilde{\mu})$ and $\phi$ is monic $\}$.

- $\Lambda(\mu, \widetilde{\mu})=\{\widetilde{\mu}(\phi) ; \phi \in \Phi(\mu, \widetilde{\mu})\}=\widetilde{\mu}(\Phi(\mu, \widetilde{\mu}))$.

- $\gamma(\mu, \widetilde{\mu})=\sup (\Lambda(\mu, \widetilde{\mu})) \in \overline{\mathbb{R}}$.

Remark 2.2. Note that if $\phi \in \Phi(\mu, \widetilde{\mu})$, then $\phi$ is an irreducible left skew polynomial and if $\mu^{\prime} \in \operatorname{Val}(R)$ with $\mu \preceq \widetilde{\mu} \preceq \mu^{\prime}$, then $d(\mu, \widetilde{\mu}) \geq d\left(\mu, \mu^{\prime}\right)$ and $d\left(\mu, \mu^{\prime}\right) \leq d\left(\widetilde{\mu}, \mu^{\prime}\right)$.

We finish this section giving a technical result.

Proposition 2.3. Let $\mu, \widetilde{\mu}, \mu^{\prime} \in \operatorname{Val}(R)$ be such that $\mu \prec \widetilde{\mu} \preceq \mu^{\prime}$, then we get the following statements:

(a) If $\mu \prec \widetilde{\mu}$, then $\mu$ is a Krull valuation on $R$.

(b) $\widetilde{\mu}(\phi)>\mu(\phi)$ for each $\phi \in \Phi\left(\mu, \mu^{\prime}\right)$. In particular, $d\left(\mu, \mu^{\prime}\right)=d(\mu, \widetilde{\mu})$ and $\Phi\left(\mu, \mu^{\prime}\right)=\Phi(\mu, \widetilde{\mu})$.

(c) Every totally ordered subset $\mathcal{S} \subset \operatorname{Val}(R)$ is bounded above.

Proof. First, assume that $\mu$ is not a Krull valuation, then $\mu^{-1}(\infty)=R f$ for some non-zero irreducible left skew polynomial $f \in R$. Note that $\mu(h)<\infty$ for each $h \in R-\{0\}$ such that $\operatorname{deg}(h)<\operatorname{deg}(f)$.

Since $\mu \neq \widetilde{\mu}$, there exists $g \in \Phi(\mu, \widetilde{\mu})$ such that $\mu(g)<\widetilde{\mu}(g)$ and $\operatorname{deg}(g)=d(\mu, \widetilde{\mu})<\operatorname{deg}(f)$. Otherwise $\operatorname{deg}(g)=d(\mu, \widetilde{\mu}) \geq \operatorname{deg}(f)$, then $g=q f+r$ with $q, r \in R$ and $\operatorname{deg}(r)<\operatorname{deg}(f) \leq$ $\operatorname{deg}(g)=d(\mu, \widetilde{\mu})$. Thus, $r \notin \widetilde{\Phi}(\mu, \widetilde{\mu})$ and $\widetilde{\mu}(g)=\widetilde{\mu}(r)=\mu(r)=\mu(g)$, which is a contradiction.

Therefore, $f=q g+r$ with $q, r \in R$ and $\operatorname{deg}(r)<\operatorname{deg}(g)=d(\mu, \widetilde{\mu})<\operatorname{deg}(f)$. In this case, also $r \notin \widetilde{\Phi}(\mu, \widetilde{\mu})$ and $\widetilde{\mu}(r)=\mu(r)=\mu(q g)<\widetilde{\mu}(q g)$. Thus, $\widetilde{\mu}(f)=\widetilde{\mu}(r) \neq \infty$, which is a contradiction, hence we have (a).

In order to show (b), let us assume $\widetilde{\mu}(\phi)=\mu(\phi)$ for some $\phi \in \Phi\left(\mu, \mu^{\prime}\right)$ and let us consider $\phi^{\prime} \in \Phi(\mu, \widetilde{\mu})$. Since $\mu\left(\phi^{\prime}\right)<\widetilde{\mu}\left(\phi^{\prime}\right) \leq \mu^{\prime}\left(\phi^{\prime}\right)$, then $d(\mu, \widetilde{\mu})=\operatorname{deg}\left(\phi^{\prime}\right) \geq \operatorname{deg}(\phi)=d\left(\mu, \mu^{\prime}\right)$. Thus, $\phi^{\prime}=q \phi+r$ with $q, r \in R$ and $\operatorname{deg}(r)<\operatorname{deg}(\phi)=d\left(\mu, \mu^{\prime}\right) \leq \operatorname{deg}\left(\phi^{\prime}\right)=d(\mu, \widetilde{\mu})$. Therefore, $\mu(r)=\widetilde{\mu}(r)=\mu^{\prime}(r)$. Moreover, since $\operatorname{deg}(q)<\operatorname{deg}\left(\phi^{\prime}\right)=d(\mu, \widetilde{\mu})$, then $\widetilde{\mu}(q)=\mu(q)$, and hence $\widetilde{\mu}(q \phi)=\mu(q \phi)$.

Since $\mu(r)=\widetilde{\mu}(r)=\mu^{\prime}(r) \geq \min \left\{\mu^{\prime}\left(\phi^{\prime}\right), \mu^{\prime}(q \phi)\right\}>\min \left\{\mu\left(\phi^{\prime}\right), \mu(q \phi)\right\}$, then $\widetilde{\mu}(r)=\mu(r)>$ $\mu\left(\phi^{\prime}\right)=\mu(q \phi)=\widetilde{\mu}(q \phi)$. Thus, we have $\widetilde{\mu}\left(\phi^{\prime}\right)=\widetilde{\mu}(q \phi)=\mu(q \phi)=\mu\left(\phi^{\prime}\right)$, which is a contradiction. Thus, $\widetilde{\mu}(\phi)>\mu(\phi)$.

Finally, to see $(\mathrm{c})$ let $\mu^{*}: R \longrightarrow \overline{\mathbb{R}}$ be given by $\mu^{*}(f)=\sup \left\{\mu_{*}(f) ; \mu_{*} \in \mathcal{S}\right\}$. Since $\mathcal{S}$ is a totally ordered set, then $\mu^{*}$ is well defined. We shall now show that $\mu^{*} \in \operatorname{Val}(R)$, and hence $\mu^{*}$ is an upper bound of $\mathcal{S}$. We only need to statements (V1) and (V2) of Definition of valuation for $\mu^{*}$. 
Let us consider $f, g \in R$. since $\widetilde{\mu}^{*}(f g)=\widetilde{\mu}^{*}(f)+\widetilde{\mu}^{*}(g) \leq \mu^{*}(f)+\mu^{*}(g)$ for all $\widetilde{\mu}^{*} \in \mathcal{S}$, then $\mu^{*}(f g) \leq \mu^{*}(f)+\mu^{*}(g)$. To see that the equality holds, assume $\mu^{*}(f g)<\mu^{*}(f)+\mu^{*}(g)$. Then there exist $\widetilde{\mu}^{\prime}, \widetilde{\mu}^{\prime \prime} \in \mathcal{S}$ such that $\mu^{*}(f)-\widetilde{\mu}^{\prime}(f)<\epsilon / 4$ and $\mu^{*}(g)-\widetilde{\mu}^{\prime \prime}(g)<\epsilon / 4$, where $\epsilon=\mu^{*}(f)+\mu^{*}(g)-\mu^{*}(f g)>0$. Since $\mathcal{S}$ is a totally ordered set, we can also assume without loss of generality $\widetilde{\mu}^{\prime} \preceq \widetilde{\mu}^{\prime \prime}$. Therefore, $\widetilde{\mu}^{\prime \prime}(f g) \leq \mu^{*}(f g)<\widetilde{\mu}^{\prime}(f)+\widetilde{\mu}^{\prime \prime}(g) \leq \widetilde{\mu}^{\prime \prime}(f)+\widetilde{\mu}^{\prime \prime}(g)=\widetilde{\mu}^{\prime \prime}(f g)$ which is a contradiction. Hence, $\mu^{*}(f g)=\mu^{*}(f)+\mu^{*}(g)$ and we get (V1).

In order to show (V2), let us assume $\min \left\{\mu^{*}(f), \mu^{*}(g)\right\}=\mu^{*}(f)$ (similarly if $\min \left\{\mu^{*}(f), \mu^{*}(g)\right\}=$ $\left.\mu^{*}(g)\right)$.

If $\mu^{*}(g) \geq \mu^{*}(f)>\mu^{*}(f+g)$, then there exist $\widetilde{\mu}^{\prime}, \widetilde{\mu}^{\prime \prime} \in \mathcal{S}$ such that $\widetilde{\mu}^{\prime}(g)>\mu^{*}(f+g) \geq \widetilde{\mu}^{\prime \prime}(f+g)$ and $\widetilde{\mu} \prime(f)>\mu^{*}(f+g) \geq \widetilde{\mu}(f+g)$. Since $\mathcal{S}$ is a totally ordered set, we can also assume without loss of generality $\widetilde{\mu}^{\prime} \preceq \widetilde{\mu}^{\prime \prime}$. Thus, $\widetilde{\mu}^{\prime \prime}(g) \geq \widetilde{\mu}^{\prime}(g)$ and $\min \left\{\widetilde{\mu}^{\prime \prime}(f), \widetilde{\mu}^{\prime \prime}(g)\right\}>\mu^{*}(f+g) \geq \widetilde{\mu}^{\prime \prime}(f+g)$ which is a contradiction. Hence, $\min \left\{\mu^{*}(f), \mu^{*}(g)\right\} \leq \mu^{*}(f+g)$ and we get (V2).

\section{Augmented and limit valuations}

We begin introducing some notation. For each $\varphi, a \in R$ we denote by $q(\varphi, a), r(\varphi, a)$ the unique elements of $R$ such that $\varphi \cdot a=q(\varphi, a) \varphi+r(\varphi, a)$ with $\operatorname{deg}(r(\varphi, a))<\operatorname{deg}(\varphi)$, i.e. the left quotient and the left rest in the left division of $\varphi \cdot a$ by $\varphi$.

Throughout this section, $\mu, \widetilde{\mu} \in \operatorname{Val}(R)$ will be two fixed real valuations such that $\mu \prec \widetilde{\mu}$. Since, $\Phi(\mu, \widetilde{\mu}) \neq \emptyset$, we also fix $\phi \in \Phi(\mu, \widetilde{\mu})$.

Next technical result relates the properties of the left division by $\phi$ with the order $\preceq$.

Lemma 3.1. With the above assumptions and notation, let $a, f \in R$ be such that $0 \leq \operatorname{deg}(a)<$ $\operatorname{deg}(\phi)<\operatorname{deg}(f)$. We have the following statements:

(a) $\widetilde{\mu}(a)=\mu(a)=\mu(q(\phi, a))=\widetilde{\mu}(q(\phi, a))<\mu(r(\phi, a))-\widetilde{\mu}(\phi)$.

(b) Let us write $\phi^{n} \cdot a=a_{n}^{(n)} \phi^{n}+a_{n-1}^{(n)} \phi^{n-1}+\cdots+a_{0}^{(n)}$ with $\operatorname{deg}(a)=\operatorname{deg}\left(a_{n}^{(n)}\right)$ and $\operatorname{deg}\left(a_{i}^{(n)}\right)<\operatorname{deg}(\phi), 0 \leq i \leq n-1$. Then $\widetilde{\mu}\left(\phi^{n} \cdot a\right)=\widetilde{\mu}\left(a_{n}^{(n)} \phi^{n}\right)<\widetilde{\mu}\left(a_{i}^{(n)} \phi^{i}\right), 0 \leq i \leq n-1$.

(c) $\mu(f) \leq \mu(r(\phi, f))$ and $\mu(f) \leq \mu(q(\phi, f) \cdot \phi)$.

Proof. First, let us assume $\mu(r(\phi, a)) \leq \mu(\phi \cdot a)=\mu(a \cdot \phi)$. Then $\widetilde{\mu}(\phi \cdot a)>\mu(\phi \cdot a) \geq \mu(r(\phi, a))=$ $\widetilde{\mu}(r(\phi, a))=\widetilde{\mu}(q(\phi, a) \phi)>\mu(q(\phi, a) \phi)$. Since $\mu(r(\phi, a)) \geq \min \{\mu(\phi \cdot a), \mu(q(\phi, a) \phi)\}$ and $\mu(\phi \cdot a)>$ $\mu(q(\phi, a) \phi)$. Therefore, $\mu(r(\phi, a))=\mu(q(\phi, a) \phi)$, which is a contradiction. So we have (a).

(b) follows easily from (a).

Finally, if either $\mu(f)>\mu(q(\phi, f) \cdot \phi)$ or $\mu(f)>\mu(r(\phi, f))$, then $\mu(f)>\mu(q(\phi, f) \cdot \phi)=\mu(r(\phi, f))$. Thus $\widetilde{\mu}(f) \geq \mu(f)>\mu(r(\phi, f))=\widetilde{\mu}(r(\phi, f))$ and $\widetilde{\mu}(q(\phi, f) \cdot \phi)>\mu(q(\phi, f) \cdot \phi)=\mu(r(\phi, f))=$ $\widetilde{\mu}(r(\phi, f))$, which is a contradiction, and this shows (c).

Since we are working with $\mathbb{R}$ as group of values, we need a compatibility property for real valuations on $R$. The next result allows us to do this.

Proposition 3.2. With the above assumptions and notation, let us denote by

$$
\mathcal{I}(\sigma, \delta, \mu, \widetilde{\mu}, \phi)=\min \{\mu(r(\phi, a))-\mu(a) ; a \in R, 0 \leq \operatorname{deg}(a)<\operatorname{deg}(\phi)\} .
$$

Then $\mathcal{I}(\sigma, \delta, \mu, \widetilde{\mu}, \phi) \in \overline{\mathbb{R}}$ and $\mathcal{I}(\sigma, \delta, \mu, \widetilde{\mu}, \phi) \geq \widetilde{\mu}(\phi)>\mu(\phi)$. Furthermore, let us consider a non negative integer $i$ and $b \in R$ such that $0 \leq \operatorname{deg}(b)<\operatorname{deg}(\phi)$. Let us write $\phi^{i} b=\sum_{k=0}^{i} b_{k}^{(i)} \phi^{k}$, with 
$\operatorname{deg}\left(b_{k}^{(i)}\right)<\operatorname{deg}(\phi), 0 \leq k \leq i$. Then $\mu\left(b_{i}^{(i)}\right)+i \gamma<\mu\left(b_{k}^{(i)}\right)+k \gamma, 0 \leq k \leq i-1$ for each $\gamma$ such that $\mu(\phi)<\gamma \leq \mathcal{I}(\sigma, \delta, \mu, \widetilde{\mu}, \phi)$.

Proof. $\mathcal{I}(\sigma, \delta, \mu, \widetilde{\mu}, \phi) \in \overline{\mathbb{R}}$ and $\widetilde{\mu}(\phi) \leq \mathcal{I}(\sigma, \delta, \mu, \widetilde{\mu}, \phi)$ follow from Lemma 3.1 (a). We proof the second statement by induction on $i$. The case $i=1$ again follows from Lemma 3.1 (a).

Therefore, assume $\mu\left(b_{l}^{(l)}\right)+l \gamma<\mu\left(b_{k}^{(l)}\right)+k \gamma$ for $0 \leq k \leq l-1$ and $1 \leq l \leq i-1$. We can write $\phi^{i} b=\phi\left(\phi^{i-1} b\right)=\phi\left(\sum_{s=0}^{i-1} b_{s}^{(i-1)} \phi^{s}\right)=\sum_{s=0}^{i-1}\left(\phi b_{s}^{(i-1)}\right) \phi^{s}$. By Lemma 3.1 (a), $\phi^{i} b=$ $\sum_{s=0}^{i-1}\left(q\left(\phi, b_{s}^{(i-1)}\right) \cdot \phi^{s+1}+r\left(\phi, b_{s}^{(i-1)}\right) \cdot \phi^{s}\right), b_{i}^{(i)}=q\left(\phi, b_{i-1}^{(i-1)}\right), b_{l}^{(i)}=q\left(\phi, b_{l-1}^{(i-1)}\right)+r\left(\phi, b_{l}^{(i-1)}\right)$, $1 \leq l \leq i-1$ and $b_{0}^{(i)}=r\left(\phi, b_{0}^{(i-1)}\right)$. Moreover, $\mu\left(b_{s}^{(i-1)}\right)=\mu\left(q\left(\phi, b_{s}^{(i-1)}\right)\right)<\mu\left(r\left(\phi, b_{s}^{(i-1)}\right)\right)-\widetilde{\mu}(\phi)$ for $0 \leq s \leq i-1$

By induction hypothesis $\mu\left(q\left(\phi, b_{l-1}^{(i-1)}\right)\right)+l \gamma=\mu\left(b_{l-1}^{(i-1)}\right)+(l-1) \gamma+\gamma>\mu\left(b_{i-1}^{(i-1)}\right)+(i-1) \gamma+\gamma$ and also $\mu\left(r\left(\phi, b_{l}^{(i-1)}\right)\right)+l \gamma>\mu\left(b_{l}^{(i-1)}\right)+\gamma+l \gamma \geq \mu\left(b_{i-1}^{(i-1)}\right)+(i-1) \gamma+\gamma$, by definition of $\mathcal{I}(\sigma, \delta, \mu, \widetilde{\mu}, \phi)$. Hence $\mu\left(b_{l}^{(i)}\right)+l \gamma=\mu\left(q\left(\phi, b_{l-1}^{(i-1)}\right)+r\left(\phi, b_{l}^{(i-1}\right)\right)+l \gamma \geq \min \left\{\mu\left(q\left(\phi, b_{l-1}^{(i-1)}\right)\right)+\right.$ $\left.l \gamma, \mu\left(r\left(\phi, b_{l}^{(i-1)}\right)\right)+l \gamma\right\}>\mu\left(b_{i-1}^{(i-1)}\right)+i \gamma=\mu\left(q\left(\phi, b_{i-1}^{(i-1)}\right)\right)+i \gamma=\mu\left(b_{i}^{(i)}\right)+i \gamma$ for $1 \leq l \leq i-1$.

To finish, $\mu\left(b_{0}^{(i)}\right)=\mu\left(r\left(\phi, b_{0}^{(i-1)}\right)\right)>\mu\left(b_{0}^{(i-1)}\right)+\gamma>\mu\left(b_{i-1}^{(i-1)}\right)+(i-1) \gamma+\gamma=\mu\left(b_{i}^{(i)}\right)+i \gamma$ by Lemma 3.1 (a) and induction hypothesis.

Definition 3.3. We shall say that $\mathcal{I}(\sigma, \delta, \mu, \widetilde{\mu}, \phi)$ is the compatibility index of $\phi$ with respect to $\mu$ and $\widetilde{\mu}$.

To define augmented valuations as in $[\mathrm{M}, \mathrm{V}]$ we first need the following result.

Theorem 3.4. With the above assumptions and notation, for each $\gamma \in \overline{\mathbb{R}}$ with $\mathcal{I}(\sigma, \delta, \mu, \widetilde{\mu}, \phi) \geq$ $\gamma>\mu(\phi)$ let us write $\mu_{\gamma}(g)=\min \left\{\mu\left(g_{i}\right)+i \gamma ; 0 \leq i \leq r\right\}$ for all $g \in R$, where $g=\sum_{i=0}^{r} g_{i} \phi^{i}$ with $\operatorname{deg}\left(g_{i}\right)<\operatorname{deg}(\phi)=d(\mu, \widetilde{\mu}), 0 \leq i \leq r$. Then $\mu_{\gamma} \in \operatorname{Val}(R), \mu \preceq \mu_{\gamma}$ and $\mu_{\gamma} \preceq \widetilde{\mu}$ when $\gamma \leq \widetilde{\mu}(\phi)$.

Proof. Let us consider $f, g \in R-\{0\}$ and write $f=\sum_{i=0}^{d} a_{i} \phi^{i}$ and $g=\sum_{j=0}^{e} b_{j} \phi^{j}$ with $0 \leq$ $\operatorname{deg}\left(a_{i}\right), \operatorname{deg}\left(b_{j}\right)<\operatorname{deg}(\phi), 0 \leq i \leq d$ and $0 \leq j \leq e$.

From an easily computation we get $\mu_{\gamma}(f+g) \geq \min \left\{\mu_{\gamma}(f), \mu_{\gamma}(g)\right\}$.

On the other hand, let us write $f g=\sum_{i=0}^{d} \sum_{j=0}^{e}\left(a_{i} \phi^{i} b_{j} \phi^{j}\right)=\sum_{0 \leq l \leq d+e} c_{l} \phi^{l}$ with $\operatorname{deg}\left(c_{l}\right)<\operatorname{deg}(\phi)$ and $\phi^{i} b_{j} \phi^{j}=\sum_{k=0}^{d+e} b_{k}^{(i, j)} \phi^{k}$ where $b_{k}^{(i, j)}=0$ for $k>i+j$ and $k<j$. Therefore, $c_{l}=\sum_{i=0}^{d} \sum_{j=0}^{e} a_{i} b_{l}^{(i, j)}$

Note that $\mu\left(a_{i}\right)+\mu\left(b_{i+j}^{(i, j)}\right)+(i+j) \gamma=\mu\left(a_{i}\right)+\mu\left(b_{j}\right)+(i+j) \gamma<\mu\left(a_{i}\right)+\mu\left(b_{k}^{(i, j)}\right)+k \gamma$ for $k \neq i+j$ and $0 \leq k \leq d+e$ by Lemma 3.1 (b) and Proposition 3.2. Hence, $\mu_{\gamma}\left(a_{i} \phi^{i} b_{j} \phi^{j}\right)=\mu\left(a_{i}\right)+\mu\left(b_{j}\right)+(i+j) \gamma$

Furthermore, $\mu_{\gamma}(f g) \geq \min \left\{\mu_{\gamma}\left(a_{i} \phi^{i} b_{j} \phi^{j}\right) ; 0 \leq i \leq d ; 0 \leq j \leq e\right\} \geq \min \left\{\mu\left(a_{i}\right)+\mu\left(b_{j}\right)+(i+\right.$ j) $\gamma ; 0 \leq i \leq d ; 0 \leq j \leq e\} \geq \min _{0 \leq i \leq d}\left\{\mu\left(a_{i}\right)+i \gamma\right\}+\min _{0 \leq j \leq e}\left\{\mu\left(b_{j}\right)+j \gamma\right\}=\mu_{\gamma}(f)+\mu_{\gamma}(g)$. 
Now, let $i_{0}$ (resp. $j_{0}$ ) be the greatest index such that $0 \leq i_{0} \leq d$ (resp. such that $0 \leq j_{0} \leq e$ ) and $\mu_{\gamma}(f)=\mu\left(a_{i_{0}}\right)+i_{0} \gamma$ (resp. $\left.\mu_{\gamma}(g)=\mu\left(b_{j_{0}}\right)+j_{0} \gamma\right)$. We have $\mu_{\gamma}(f)<\mu\left(a_{i}\right)+i \gamma$ (resp. $\left.\mu_{\gamma}(g)<\mu\left(b_{j}\right)+j \gamma\right)$ for $d \geq i>i_{0}\left(\right.$ resp. $\left.e \geq j>j_{0}\right)$.

Let $(i, j) \neq\left(i_{0}, j_{0}\right)$ be such that $b_{i_{0}+j_{0}}^{(i, j)} \neq 0$. Then $i>i_{0}$ or $j>j_{0}$. (Note that $j \leq i_{0}+j_{0} \leq i+j$.) Moreover, $\mu\left(b_{i_{0}+j_{0}}^{(i, j)}\right) \geq \mu\left(b_{i+j}^{(i, j)}\right)=\mu\left(b_{j}\right) \geq \mu\left(b_{j_{0}}\right)$ and $\mu\left(a_{i}\right) \geq \mu\left(a_{i_{0}}\right)$.

Thus, if $i>i_{0}$, then $\mu\left(a_{i}\right)>\mu\left(a_{i_{0}}\right)$ and $\mu\left(a_{i} b_{i_{0}+j_{0}}^{(i, j)}\right)>\mu\left(a_{i_{0}}\right)+\mu\left(b_{j_{0}}\right)$ and also if $j>j_{0}$, then $\mu\left(b_{j}\right)>\mu\left(b_{j_{0}}\right)$ and $\mu\left(a_{i} b_{i_{0}+j_{0}}^{(i, j)}\right)>\mu\left(a_{i_{0}}\right)+\mu\left(b_{j_{0}}\right)$. Therefore, $\mu\left(c_{i_{0}+j_{0}}\right)=\mu\left(a_{i_{0}}\right)+\mu\left(b_{j_{0}}\right)$ and $\mu_{\gamma}(f)+\mu_{\gamma}(g) \geq \mu_{\gamma}(f g)$. Hence, $\mu_{\gamma}(f)+\mu_{\gamma}(g)=\mu_{\gamma}(f g)$.

Finally, we get $\mu(f) \leq \mu_{\gamma}(f)$ by induction on $d$. If $d=0$, then $\mu_{\gamma}(f)=\mu(f)$ and since $\mu(f) \leq \min \left\{\mu(q \phi), \mu\left(a_{0}\right)\right\}$, where $q=\sum_{i=1}^{d} a_{i} \phi^{i-1}$, then the induction step follows from Lemma 3.1 (c). Hence, since $\mu(\phi)<\mu_{\gamma}(\phi)$, we get $\mu \prec \mu_{\gamma}$.

The last assertion is straightforward.

As in $[\mathrm{M}, \mathrm{V}]$, we say that $\mu_{\gamma}$ is an augmented valuation of $\mu$ and it is denoted by $\mu_{\gamma}=$ $\left[\mu ; \mu_{\gamma}(\phi)=\gamma\right]$.

Next, we state more properties of augmented valuations.

Proposition 3.5. Under the assumptions and notation of Theorem 3.4, we have the following statements

(a) Let $\mu^{\prime} \in \operatorname{Val}(R)$ be such that $\mu \prec \mu^{\prime} \preceq \mu_{\gamma}$ and let us write $\mu^{\prime}(\phi)=\gamma^{\prime}$ and $\mu_{\gamma^{\prime}}=$ $\left[\mu ; \mu_{\gamma^{\prime}}(\phi)=\gamma^{\prime}\right]$. Then $\mu^{\prime}=\mu_{\gamma^{\prime}}$. In particular, $d\left(\mu^{\prime}, \mu_{\gamma}\right)=d\left(\mu, \mu_{\gamma}\right)$ when $\mu^{\prime} \neq \mu_{\gamma}$

(b) Let $\phi^{\prime} \in \Phi(\mu, \widetilde{\mu})$ be such that $\mu\left(\phi^{\prime}\right)<\gamma^{*} \leq \widetilde{\mu}\left(\phi^{\prime}\right)$ and write $\mu_{\gamma^{*}}=\left[\mu ; \mu_{\gamma^{*}}\left(\phi^{\prime}\right)=\gamma^{*}\right]$. If $\gamma \leq \widetilde{\mu}(\phi)$ and $\gamma \leq \gamma^{*}$, then $\mu_{\gamma}(\phi) \leq \mu_{\gamma^{*}}(\phi)$ and $\mu_{\gamma} \preceq \mu_{\gamma^{*}}$. Moreover, $\mu_{\gamma}=\mu_{\gamma^{*}}$ if and only if $\gamma=\gamma^{*}$.

(c) Let us also assume $\widetilde{\mu}(\phi)=\gamma=\gamma(\mu, \widetilde{\mu}) \in \Lambda(\mu, \widetilde{\mu})$ and $\mu_{\gamma} \neq \widetilde{\mu}$. Then $d\left(\mu_{\gamma}, \widetilde{\mu}\right)>d(\mu, \widetilde{\mu})$.

Proof. To see (a), we point out that $\mu_{\gamma^{\prime}} \preceq \mu^{\prime}$. Moreover, if $\gamma=\gamma^{\prime}$, then $\mu_{\gamma^{\prime}}=\mu^{\prime}=\mu_{\gamma}$.

Therefore, assume $\gamma^{\prime}<\gamma$ and $\mu_{\gamma^{\prime}} \prec \mu^{\prime}$. By Proposition $2.3(\mathrm{~b}), \phi \in \Phi\left(\mu_{\gamma^{\prime}}, \mu_{\gamma}\right)=\Phi\left(\mu_{\gamma^{\prime}}, \mu^{\prime}\right)$ and $\mu_{\gamma^{\prime}}(\phi)<\mu^{\prime}(\phi)$, which is a contradiction, and this proves (a).

In order to show (b), let us write $\phi=\phi^{\prime}+r$ with $r \in R$ and $\operatorname{deg}(r)<\operatorname{deg}(\phi)=\operatorname{deg}\left(\phi^{\prime}\right)$. If $\mu_{\gamma}(\phi)>\mu_{\gamma^{*}}(\phi)$, then $\gamma^{*}=\mu_{\gamma^{*}}\left(\phi^{\prime}\right) \geq \gamma=\mu_{\gamma}(\phi)>\mu_{\gamma^{*}}(\phi)=\mu(r)=\widetilde{\mu}(r)$. Therefore, $\widetilde{\mu}(\phi) \geq \mu_{\gamma}(\phi)>\widetilde{\mu}(r)$ and $\widetilde{\mu}\left(\phi^{\prime}\right) \geq \mu_{\gamma^{*}}\left(\phi^{\prime}\right)>\widetilde{\mu}(r)$. So, we have $\widetilde{\mu}(\phi), \widetilde{\mu}\left(\phi^{\prime}\right)>\widetilde{\mu}(r)$, which is a contradiction. Hence, $\mu_{\gamma}(\phi) \leq \mu_{\gamma^{*}}(\phi)$ and $\mu_{\gamma} \preceq \mu_{\gamma^{*}}$.

The last claims of (b) follow easily from (a) and the definition of augmented valuation.

For $(\mathrm{c})$, since $\mu_{\gamma} \neq \widetilde{\mu}$, there exists $\phi^{\prime} \in \Phi\left(\mu_{\gamma}, \widetilde{\mu}\right)$ such that $\mu\left(\phi^{\prime}\right) \leq \mu_{\gamma}\left(\phi^{\prime}\right)<\widetilde{\mu}\left(\phi^{\prime}\right)$ and $\operatorname{deg}\left(\phi^{\prime}\right)=$ $d\left(\mu_{\gamma}, \widetilde{\mu}\right) \geq d(\mu, \widetilde{\mu})$ (see Remark 2.2).

Let us assume $d\left(\mu_{\gamma}, \widetilde{\mu}\right)=d(\mu, \widetilde{\mu})$, then $\widetilde{\mu}\left(\phi^{\prime}\right) \leq \gamma(\mu, \widetilde{\mu})=\widetilde{\mu}(\phi)=\mu_{\gamma}(\phi)=\gamma$. Let us write $\phi=\phi^{\prime}+r$ with $r \in R$ and $\operatorname{deg}(r)<d\left(\mu_{\gamma}, \widetilde{\mu}\right)=d(\mu, \widetilde{\mu})=\operatorname{deg}(\phi)$. Since $\mu_{\gamma}\left(\phi^{\prime}\right)<\widetilde{\mu}\left(\phi^{\prime}\right) \leq$ $\mu_{\gamma}(\phi)=\widetilde{\mu}(\phi)$, then $\mu_{\gamma}(r)=\widetilde{\mu}(r)=\mu_{\gamma}\left(\phi^{\prime}\right)<\widetilde{\mu}\left(\phi^{\prime}\right) \leq \widetilde{\mu}(\phi)$. So, $\widetilde{\mu}(r)<\widetilde{\mu}\left(\phi^{\prime}\right) \leq \widetilde{\mu}(\phi)$ which is a contradiction. Hence, $d\left(\mu_{\gamma}, \widetilde{\mu}\right)>d(\mu, \widetilde{\mu})$. 
We point out that if $\gamma=\gamma(\mu, \widetilde{\mu}) \in \Lambda(\mu, \widetilde{\mu})$ we can define an augmented valuation $\mu_{\gamma}$ with $d\left(\mu_{\gamma}, \widetilde{\mu}\right)>d(\mu, \widetilde{\mu})$ (see Proposition $3.5(\mathrm{c})$ ).

The question is: What does happen when $\gamma(\mu, \widetilde{\mu}) \notin \Lambda(\mu, \widetilde{\mu})$ ? Therefore, let us assume $\gamma(\mu, \widetilde{\mu}) \notin$ $\Lambda(\mu, \widetilde{\mu})$. For each $\gamma \in \Lambda(\mu, \widetilde{\mu})$, we fix $\phi_{\gamma} \in \Phi(\mu, \widetilde{\mu})$ such that $\widetilde{\mu}\left(\phi_{\gamma}\right)=\gamma$ and we write $\mu_{\gamma}=$ $\left[\mu ; \mu_{\gamma}\left(\phi_{\gamma}\right)=\gamma\right]$.

Lemma 3.6. With the above assumptions and notation, $\mu_{\gamma}$ does not depend on $\phi_{\gamma} \in \Phi(\mu, \widetilde{\mu})$ with $\widetilde{\mu}\left(\phi_{\gamma}\right)=\gamma$ for all $\gamma \in \Lambda(\mu, \widetilde{\mu})$. Moreover, if $\gamma \leq \gamma^{\prime}$, then $\mu_{\gamma} \preceq \mu_{\gamma^{\prime}}$. In particular, $\left\{\mu_{\gamma} ; \gamma \in \Lambda(\mu, \widetilde{\mu})\right\}$ is a totally ordered subset of $\operatorname{Val}(R)$.

Proof. Let $\phi^{\prime} \in \Phi(\mu, \widetilde{\mu})$ be such that $\widetilde{\mu}\left(\phi^{\prime}\right)=\widetilde{\mu}\left(\phi_{\gamma}\right)=\gamma$. We write $\mu^{\prime}=\left[\mu ; \mu^{\prime}\left(\phi^{\prime}\right)=\gamma\right]$ and $\phi^{\prime}=$ $\phi_{\gamma}+r$ with $\operatorname{deg}(r)<\operatorname{deg}\left(\phi_{\gamma}\right)=\operatorname{deg}\left(\phi^{\prime}\right)=d(\mu, \widetilde{\mu})$. Therefore, $\widetilde{\mu}(r)=\mu(r)=\mu_{\gamma}(r)=\mu^{\prime}(r) \geq \gamma$ and $\mu_{\gamma}\left(\phi^{\prime}\right) \geq \gamma$.

For each $f \in R$, we write $f=a_{d}\left(\phi^{\prime}\right)^{d}+a_{d-1}\left(\phi^{\prime}\right)^{d-1}+\cdots+a_{0}$ with $a_{i} \in R$ and $\operatorname{deg}\left(a_{i}\right)<$ $\operatorname{deg}\left(\phi^{\prime}\right)=d(\mu, \widetilde{\mu}), 0 \leq i \leq d$. We have

$$
\mu_{\gamma}(f) \geq \min \left\{\mu_{\gamma}\left(a_{i}\left(\phi^{\prime}\right)^{i}\right) ; 0 \leq i \leq d\right\} \geq \min \left\{\mu\left(a_{i}\right)+i \gamma_{;} 0 \leq i \leq d\right\}=\mu^{\prime}(f) .
$$

Note that $\mu_{\gamma}\left(a_{i}\left(\phi^{\prime}\right)^{i}\right)=\mu\left(a_{i}\right)+i \mu_{\gamma}\left(\phi^{\prime}\right) \geq \mu\left(a_{i}\right)+i \gamma, 0 \leq i \leq d$. Hence, $\mu^{\prime} \preceq \mu_{\gamma}$. Similarly $\mu_{\gamma} \preceq \mu^{\prime}$. Thus, $\mu^{\prime}=\mu_{\gamma}$.

On the other hand, let $\gamma, \gamma^{\prime} \in \Lambda(\mu, \widetilde{\mu})$ be such that $\gamma \leq \gamma^{\prime}$ and $\phi_{\gamma}, \phi_{\gamma^{\prime}} \in \Phi(\mu, \widetilde{\mu})$ be such that $\mu_{\gamma}=\left[\mu ; \mu_{\gamma}\left(\phi_{\gamma}\right)=\gamma\right]$ and $\mu_{\gamma^{\prime}}=\left[\mu ; \mu_{\gamma^{\prime}}\left(\phi_{\gamma^{\prime}}\right)=\gamma^{\prime}\right]$. We write $\phi_{\gamma^{\prime}}=\phi_{\gamma}+r$ with $r \in R$ and $\operatorname{deg}(r)<d(\mu, \widetilde{\mu})=\operatorname{deg}\left(\phi_{\gamma}\right)=\operatorname{deg}\left(\phi_{\gamma^{\prime}}\right)$. Since $\widetilde{\mu}\left(\phi_{\gamma^{\prime}}\right)=\mu_{\gamma^{\prime}}\left(\phi_{\gamma^{\prime}}\right)=\gamma^{\prime} \geq \gamma=\mu_{\gamma}\left(\phi_{\gamma}\right)=\widetilde{\mu}\left(\phi_{\gamma}\right)$, then $\mu_{\gamma}(r)=\widetilde{\mu}(r) \geq \gamma$. Hence, $\mu_{\gamma^{\prime}}\left(\phi_{\gamma}\right) \geq \mu_{\gamma}\left(\phi_{\gamma}\right)=\gamma$ and since $\mu_{\gamma}$ is an augmented valuation, we get $\mu_{\gamma} \preceq \mu_{\gamma^{\prime}}$.

Under the assumptions and notation as in the proof of Lemma 3.6, we have $\mu_{\gamma}=\left[\mu ; \mu_{\gamma}\left(\phi_{\gamma^{\prime}}\right)=\gamma\right]$ and $\mu_{\gamma^{\prime}}=\left[\mu_{\gamma} ; \mu_{\gamma^{\prime}}\left(\phi_{\gamma^{\prime}}\right)=\gamma^{\prime}\right]$ for $\gamma, \gamma^{\prime} \in \Lambda(\mu, \widetilde{\mu})$ with $\gamma<\gamma^{\prime}$ (see Proposition 3.5 (a)).

We define $\mu_{\infty}: R \longrightarrow \overline{\mathbb{R}}$ by $\mu_{\infty}(f)=\sup \left\{\mu_{\gamma}(f) ; \gamma \in \Lambda(\mu, \widetilde{\mu})\right\}$ for each $f \in R$. From Proposition 2.3 (c) and Lemma 3.6, we have $\mu_{\infty} \in \operatorname{Val}(R)$ and $\mu \preceq \mu_{\gamma} \preceq \mu_{\infty} \preceq \widetilde{\mu}$. As in [M], $\mu_{\infty}$ is called the limit valuation for the pair $(\mu, \widetilde{\mu})$.

Now, we give the main properties of limit valuations in the following results.

Proposition 3.7. With the above assumptions and notation, let us consider $\mu^{\prime} \in \operatorname{Val}(R)$ such that $\mu \prec \mu^{\prime} \prec \mu_{\infty} \preceq \widetilde{\mu}$. Then there exists $\phi \in \Phi(\mu, \widetilde{\mu})$ such that $\gamma^{\prime}=\mu^{\prime}(\phi)<\mu_{\infty}(\phi)$ and $\mu^{\prime}=\mu_{\gamma^{\prime}}$, where $\mu_{\gamma^{\prime}}=\left[\mu ; \mu_{\gamma^{\prime}}(\phi)=\gamma^{\prime}\right]$.

Proof. If $\gamma^{\prime}=\mu^{\prime}(\phi)=\mu_{\infty}(\phi)$ for all $\phi \in \Phi(\mu, \widetilde{\mu})$, then $\mu_{\gamma} \preceq \mu^{\prime}$ for each $\gamma \in \Lambda(\mu, \widetilde{\mu})$. Thus, by definition of the limit valuation $\mu_{\infty}$, we have $\mu_{\infty} \preceq \mu^{\prime}$.

Therefore, assume $\gamma^{\prime}=\mu^{\prime}(\phi)<\mu_{\infty}(\phi)$ for some $\phi \in \Phi(\mu, \widetilde{\mu})$. If $\mu_{\gamma^{\prime}} \prec \mu^{\prime}$, then $\phi \in \Phi\left(\mu_{\gamma^{\prime}}, \mu_{\infty}\right)=$ $\Phi\left(\mu_{\gamma^{\prime}}, \mu^{\prime}\right)$ (see Proposition $\left.2.3(\mathrm{~b})\right)$ and $\gamma^{\prime}=\mu_{\gamma^{\prime}}(\phi)<\mu^{\prime}(\phi)=\gamma^{\prime}$, which is a contradiction.

Remark 3.8. Let us consider $\mu^{\prime} \in \operatorname{Val}(R)$ be such that $\mu \prec \mu^{\prime} \prec \widetilde{\mu}$ and write $\mu_{*}=\mu_{\infty}$, when $\gamma(\mu, \widetilde{\mu}) \notin \Lambda(\mu, \widetilde{\mu})$ and $\mu_{*}=\left[\mu ; \mu_{*}(\phi)=\gamma(\mu, \widetilde{\mu})\right]$, when $\gamma(\mu, \widetilde{\mu}) \in \Lambda(\mu, \widetilde{\mu})$ for some $\phi \in \Phi(\mu, \widetilde{\mu})$ with $\widetilde{\mu}(\phi)=\gamma(\mu, \widetilde{\mu})$. Then we have the following possibilities:

(1) $\mu^{\prime}\left(\phi^{\prime}\right)=\widetilde{\mu}\left(\phi^{\prime}\right)$ for each $\phi^{\prime} \in \Phi(\mu, \widetilde{\mu})$. In this case, $\mu_{*} \preceq \mu^{\prime}$. (This follows clearly from the definition of $\mu_{*}$.) 
(2) There exists $\phi^{\prime} \in \Phi(\mu, \widetilde{\mu})$ such that $\gamma^{\prime}=\mu^{\prime}\left(\phi^{\prime}\right)<\widetilde{\mu}\left(\phi^{\prime}\right)$. In this case, $\mu_{\gamma^{\prime}}=\mu^{\prime} \prec \mu_{*}$, where $\mu_{\gamma^{\prime}}=\left[\mu ; \mu_{*}\left(\phi^{\prime}\right)=\gamma^{\prime}\right]$. (Note that $\mu_{\gamma^{\prime}} \preceq \mu^{\prime}$ and if $\mu_{\gamma^{\prime}} \prec \mu^{\prime}$, then $\phi^{\prime} \in \Phi\left(\mu_{\gamma^{\prime}}, \widetilde{\mu}\right)=$ $\Phi\left(\mu_{\gamma^{\prime}}, \mu^{\prime}\right)$ and $\gamma^{\prime}=\mu_{\gamma^{\prime}}\left(\phi^{\prime}\right)<\mu^{\prime}\left(\phi^{\prime}\right)=\gamma^{\prime}$ which is a contradiction. Moreover, if $\mu_{*}=\mu_{\infty}$ then $\mu_{\gamma^{\prime}} \prec \mu_{*}$ and if $\mu_{*}=\left[\mu ; \mu_{*}(\phi)=\gamma(\mu, \widetilde{\mu})\right]$, then $\mu_{\gamma^{\prime}} \prec \mu_{*}$ by Proposition 3.5 (b).)

Proposition 3.9. With the above assumptions and notation, we have:

(1) If $\mu_{\infty}(f)=\infty$ for some non-zero polynomial $f \in R$, then $\mu_{\infty}=\widetilde{\mu}$.

(2) If $\gamma(\mu, \widetilde{\mu})=\infty$, then $\mu_{\infty}=\widetilde{\mu}$.

(3) If $\mu_{\infty} \neq \widetilde{\mu}$, then $\mu_{\infty}$ is a Krull valuation and $\gamma(\mu, \widetilde{\mu})<\infty$. In this case, $d\left(\mu_{\infty}, \widetilde{\mu}\right)>$ $d(\mu, \widetilde{\mu})$.

Proof. Assume $\mu_{\infty}(f)=\infty$ for some $f \in R-\{0\}$. If $\mu_{\infty} \prec \widetilde{\mu}$, then $\mu_{\infty}$ is a Krull valuation by Proposition 2.3 (a), which is a contradiction. Hence, (1) is showed.

To see (2), assume $\gamma(\mu, \widetilde{\mu})=\infty$ and $\mu_{\infty} \neq \widetilde{\mu}$. There exists $g \in \Phi\left(\mu_{\infty}, \widetilde{\mu}\right)$ such that $\mu_{\infty}(g)<\widetilde{\mu}(g)$. In particular, $\mu_{\infty}(g)<\infty$ and $d(\mu, \widetilde{\mu}) \leq d\left(\mu_{\infty}, \widetilde{\mu}\right)$ (note that $\mu \preceq \mu_{\infty} \preceq \widetilde{\mu}$ ).

Since $\gamma(\mu, \widetilde{\mu})=\infty$, let us consider $\gamma \in \Lambda(\mu, \widetilde{\mu})$ with

$$
\left(d\left(\mu_{\infty}, \widetilde{\mu}\right)-d(\mu, \widetilde{\mu})\right) \mu_{\infty}(T)+\gamma>\mu_{\infty}(g)
$$

and write $h=g-T^{d\left(\mu_{\infty}, \widetilde{\mu}\right)-d(\mu, \widetilde{\mu})} \phi_{\gamma} . \quad$ As $\operatorname{deg}(g)=d\left(\mu_{\infty}, \widetilde{\mu}\right)$ and $\operatorname{deg}\left(\phi_{\gamma}\right)=d(\mu, \widetilde{\mu})$, then $\operatorname{deg}(h)<\operatorname{deg}(g)=d\left(\mu_{\infty}, \widetilde{\mu}\right)$. Furthermore, $\mu_{\gamma} \preceq \mu_{\infty} \preceq \widetilde{\mu}$ and $\gamma=\mu_{\gamma}\left(\phi_{\gamma}\right)=\mu_{\infty}\left(\phi_{\gamma}\right)=\widetilde{\mu}\left(\phi_{\gamma}\right)$. Thus, $\mu_{\infty}\left(T^{d\left(\mu_{\infty}, \widetilde{\mu}\right)-d(\mu, \widetilde{\mu})} \phi_{\gamma}\right)>\mu_{\infty}(g)$ and $\mu_{\infty}(h)=\mu_{\infty}(g)$.

On the other hand, since $\widetilde{\mu}\left(T^{d\left(\mu_{\infty}, \widetilde{\mu}\right)-d(\mu, \widetilde{\mu})} \phi_{\gamma}\right) \geq \mu_{\infty}\left(T^{d\left(\mu_{\infty}, \widetilde{\mu}\right)-d(\mu, \widetilde{\mu})} \phi_{\gamma}\right)>\mu_{\infty}(g)$, then $\widetilde{\mu}(h) \geq$ $\min \left\{\widetilde{\mu}(g), \widetilde{\mu}\left(T^{d\left(\mu_{\infty}, \widetilde{\mu}\right)-d(\mu, \widetilde{\mu})} \phi_{\gamma}\right)\right\}>\mu_{\infty}(g)=\mu_{\infty}(h)$ which contradicts $\operatorname{deg}(h)<d\left(\mu_{\infty}, \widetilde{\mu}\right)$.

Finally, if $\mu_{\infty} \neq \widetilde{\mu}$, then $\mu_{\infty}$ is a Krull valuation by Proposition 2.3 (a) and $\gamma(\mu, \widetilde{\mu})<\infty$ by (2). Moreover, since $\mu \preceq \mu_{\infty} \preceq \widetilde{\mu}$, then $d(\mu, \widetilde{\mu}) \leq d\left(\mu_{\infty}, \widetilde{\mu}\right)$.

Assume $d(\mu, \widetilde{\mu})=d\left(\mu_{\infty}, \widetilde{\mu}\right)$, then $\mu(\phi) \leq \mu_{\infty}(\phi)<\widetilde{\mu}(\phi)$ for each $\phi \in \Phi\left(\mu_{\infty}, \widetilde{\mu}\right)$. In particular, $\widetilde{\mu}(\phi) \in \Lambda(\mu, \widetilde{\mu})$. Since $\gamma(\mu, \widetilde{\mu}) \notin \Lambda(\mu, \widetilde{\mu})$, there exists $\gamma_{0} \in \Lambda(\mu, \widetilde{\mu})$ such that $\gamma>\widetilde{\mu}(\phi)$ for each $\gamma \in \Lambda(\mu, \widetilde{\mu})$ with $\gamma \geq \gamma_{0}$. Therefore, we can write $\phi=\phi_{\gamma}+r_{\gamma}$ with $r_{\gamma} \in R$ and $\operatorname{deg}\left(r_{\gamma}\right)<$ $\operatorname{deg}\left(\phi_{\gamma}\right)=d(\mu, \widetilde{\mu})=d\left(\mu_{\infty}, \widetilde{\mu}\right)=\operatorname{deg}(\phi)$. Since $\mu_{\infty}\left(\phi_{\gamma}\right) \geq \mu_{\gamma}\left(\phi_{\gamma}\right)=\gamma=\widetilde{\mu}\left(\phi_{\gamma}\right)>\widetilde{\mu}(\phi)$, then $\widetilde{\mu}(\phi)=\widetilde{\mu}\left(r_{\gamma}\right)=\mu_{\infty}\left(r_{\gamma}\right)=\mu_{\infty}(\phi)$, which contradicts $\phi \in \Phi\left(\mu_{\infty}, \widetilde{\mu}\right)$. This completes the proof of (3).

To finish this section, we shall give an useful technical result on augmented valuations.

Lemma 3.10. With the above assumptions and notation, let us also assume $\gamma \leq \widetilde{\mu}(\phi)$ (i.e. $\left.\mu_{\gamma} \preceq \widetilde{\mu}\right)$. Then $\operatorname{deg}(\phi) \cdot \widetilde{\mu}\left(\phi^{\prime}\right) \geq \operatorname{deg}\left(\phi^{\prime}\right) \cdot \gamma$ for each $\mu^{\prime} \in \operatorname{Val}(R)$ with $\widetilde{\mu} \preceq \mu^{\prime}$ and each $\phi^{\prime} \in \Phi\left(\widetilde{\mu}, \mu^{\prime}\right)$.

Proof. Let us write $\operatorname{deg}(\phi)=d$ and $\operatorname{deg}\left(\phi^{\prime}\right)=d^{\prime}$ and assume $d \cdot \widetilde{\mu}\left(\phi^{\prime}\right)<d^{\prime} \cdot \gamma$. Let $\beta \in \mathbb{R}$ be such that $d \cdot \widetilde{\mu}\left(\phi^{\prime}\right)<d \cdot \beta<d^{\prime} \cdot \gamma$ and let us write $\widetilde{\mu}_{\beta}=\left[\widetilde{\mu} ; \widetilde{\mu}_{\beta}\left(\phi^{\prime}\right)=\beta\right]$.

We can write $\phi^{d^{\prime}}=\left(\phi^{\prime}\right)^{d}+a_{1}\left(\phi^{\prime}\right)^{d-1}+\cdots+a_{s}$, with $a_{i} \in R$ and $\operatorname{deg}\left(a_{i}\right)<d^{\prime}, 1 \leq i \leq s$. Therefore, $\operatorname{deg}\left(\phi^{\prime}\right) \cdot \gamma=\mu_{\gamma}\left(\phi^{d^{\prime}}\right) \leq \widetilde{\mu}_{\beta}\left(\phi^{d^{\prime}}\right) \leq \widetilde{\mu}_{\beta}\left(\left(\phi^{\prime}\right)^{d}\right)=d \cdot \beta<d^{\prime} \cdot \gamma$ which is a contradiction. Hence, $\operatorname{deg}(\phi) \cdot \widetilde{\mu}\left(\phi^{\prime}\right) \geq \operatorname{deg}\left(\phi^{\prime}\right) \cdot \gamma$. 


\section{INVARIANTS}

Throughout this section, $\mu \in \operatorname{Val}(R)$ will be a fixed real valuation. Our main objective is to introduce two invariants for such a $\mu$, the Apéry base and the iterated sequence associated with $\mu$, and study the relations between them.

For each non-negative integer $i \geq 1$, we define

$$
\omega_{i}(\mu)=\sup \{\mu(f) ; f \in R, \text { monic with } \operatorname{deg}(f)=i\} \in \overline{\mathbb{R}}
$$

Note that $\omega_{i}(\mu)+\omega_{j}(\mu) \leq \omega_{i+j}(\mu)$ and if $\omega_{i}(\mu)=\infty$ for some non negative integer $i$, then $\omega_{j}(\mu)=\infty$ for all $j \geq i$. We shall say that $\left\{\omega_{i}(\mu)\right\}_{i \geq 0}$ is the Apéry base of $\mu$, where $\omega_{0}(\mu)=$ $\mu(T)$.

Next, we define inductively the iterated sequence of valuations $\left\{\left(\mu_{i}, d_{i}, \gamma_{i}\right)\right\}_{i=0}^{t}$ associated with $\mu$.

First, we denote by $\mu_{0}\left(\sum_{0 \leq i \leq d} a_{i} T^{i}\right)=\min \left\{\mu\left(a_{i}\right)+i \mu(T) ; 0 \leq i \leq d\right\}$. Since the restriction $\mu_{\mid D}$ of $\mu$ to $D$ is $\mu(T)$-compatible (in the sense of [CZ]), then $\mu_{0} \in \operatorname{Val}(R)$ by Proposition 4.5 of [CZ]. We write $d_{0}=1$ and $\gamma_{0}=\mu(T)$. Note that $\mu_{0} \preceq \mu$ and possibly $\mu(T)<0$.

Since $\left(\mu_{0}, d_{0}, \gamma_{0}\right)$ is already defined, let us assume that $\left(\mu_{s}, d_{s}, \gamma_{s}\right)$ is defined for some $s \geq 0$ with $\mu_{s} \preceq \mu$. We have the following possibilities:

a) If $\mu_{s}=\mu$, then $t=s$ and there is nothing to do.

b) If $\mu_{s} \neq \mu$, then $t>s$ and we have two possibilities more:

b.1) $\gamma\left(\mu_{s}, \mu\right) \in \Lambda\left(\mu_{s}, \mu\right)$. In this case, $d_{s+1}=d\left(\mu_{s}, \mu\right), \gamma_{s+1}=\gamma\left(\mu_{s}, \mu\right)$ and $\mu_{s+1}=$ $\left[\mu_{s} ; \mu_{s+1}\left(\phi_{s+1}\right)=\gamma_{s+1}\right]$, where $\phi_{s+1} \in \Phi\left(\mu_{s}, \mu\right)$ with $\mu\left(\phi_{s+1}\right)=\gamma_{s+1}=\gamma\left(\mu_{s}, \mu\right)$; i.e. $\mu_{s+1}$ is an augmented valuation of $\mu_{s}$.

b.2) $\gamma\left(\mu_{s}, \mu\right) \notin \Lambda\left(\mu_{s}, \mu\right)$. In this case, $d_{s+1}=d\left(\mu_{s}, \mu\right), \gamma_{s+1}=\gamma\left(\mu_{s}, \mu\right)$ and $\mu_{s+1}$ is the limit valuation associated with the pair $\left(\mu_{s}, \mu\right)$.

The existence of the iterated sequence of valuations associated with $\mu$ follows from Propositions 3.5 (c) and 3.9 and the following statements follow from the results of Section 3.

- $t \in \mathbb{N} \cup\{\infty\}$ and if $t \in \mathbb{N}$, then $\mu_{t}=\mu$.

- $\mu_{i}$ is a Krull valuation on $R, i<t$.

- $\mu_{i-1} \prec \mu_{i} \preceq \mu, 1 \leq i \leq t$.

- $d_{0}=1$ and $d_{i}<d_{i+1}, 1 \leq i<t$.

- $\gamma_{0}=\mu(T), \gamma_{i} \in \mathbb{R}, 1 \leq i<t$ and $\gamma_{t} \in \overline{\mathbb{R}}$ when $t \in \mathbb{N}$.

Firstly, we study the behavior of the iterated sequence of valuations associated with $\mu$ respect to the order relation $\preceq$.

Proposition 4.1. With the above assumptions and notation, let $\mu^{\prime} \in \operatorname{Val}(R)$ be such that $\mu^{\prime} \prec \mu$ and let $\left\{\left(\mu_{i}^{\prime}, d_{i}^{\prime}, \gamma_{i}^{\prime}\right)\right\}_{i=0}^{t^{\prime}}$ be the iterated sequence of valuations associated with $\mu^{\prime}$. We have the following possibilities:

a) $\mu^{\prime}(T)<\mu(T)$. In this case, $t^{\prime}=0$ and $\mu_{0}^{\prime}=\mu^{\prime} \prec \mu_{0}$.

b) $\mu^{\prime}(T)=\mu(T)$. In this case, there exists a non-negative integer $1 \leq l \leq t$ such that $\mu_{l-1} \preceq \mu^{\prime} \prec \mu_{l}$. Furthermore, either $\mu_{l-1}=\mu^{\prime}, t^{\prime}=l-1, \mu_{i}^{\prime}=\mu_{i}, d_{i}^{\prime}=d_{i}$ and $\gamma_{i}^{\prime}=\gamma_{i}$, 
$0 \leq i \leq t^{\prime}=l-1$; or $\mu_{l-1} \prec \mu^{\prime}, t^{\prime}=l, \mu_{i}^{\prime}=\mu_{i}, d_{i}^{\prime}=d_{i}, \gamma_{i}^{\prime}=\gamma_{i}$ for $0 \leq i \leq t^{\prime}-1=l-1$, $d_{t^{\prime}}^{\prime}=\bar{d}_{t^{\prime}}$ and there exists $\phi \in \Phi\left(\mu_{l-1}, \mu\right)$ such that $\gamma_{t^{\prime}}^{\prime}=\mu^{\prime}(\phi)<\mu(\phi)$ and $\mu^{\prime}=\mu_{t^{\prime}}=$ $\left[\mu_{l-1} ; \mu_{t^{\prime}}(\phi)=\gamma_{t^{\prime}}^{\prime}\right]$.

In particular, $\mathcal{J}_{\mu}=\left\{\mu^{\prime} \in \operatorname{Val}(R) ; \mu^{\prime} \preceq \mu\right\}$ is a totally ordered subset of $\operatorname{Val}(R)$.

Proof. First, assume $\mu^{\prime}(T)<\mu(T)$. Thus, $\mu_{0}^{\prime} \preceq \mu^{\prime} \prec \mu$. If $\mu_{0}^{\prime} \prec \mu^{\prime}$, then $\Phi\left(\mu_{0}^{\prime}, \mu^{\prime}\right)=\Phi\left(\mu_{0}^{\prime}, \mu\right)$ (see Proposition $2.3(\mathrm{~b})$ ), and since $\mu_{0}^{\prime}(T)=\mu^{\prime}(T)<\mu(T)$, then $T \in \Phi\left(\mu_{0}^{\prime}, \mu^{\prime}\right)$ which is a contradiction. Hence, $\mu_{0}^{\prime}=\mu^{\prime} \prec \mu_{0}$ and this shows a).

Now, assume $\mu^{\prime}(T)=\mu(T)$. Thus, $\mu_{0}^{\prime}=\mu_{0} \preceq \mu^{\prime}$. Let $l \in \mathbb{N} \cup\{\infty\}$ be the greatest non negative integer or infinity such that $\mu_{j} \preceq \mu^{\prime}$ for $0 \leq j<l$. We point out that $l \neq \infty$. If $l=\infty$, then $t=\infty$ and since $\left\{d_{i}\right\}_{i=1}^{\infty}$ is a strictly increasing sequence of non-negative integers, then for every $f \in R$ there exists $j \geq 1$ with $\operatorname{deg}(f)<d_{j}$ and $\mu(f)=\mu_{j}(f) \leq \mu^{\prime}(f)$. So, $\mu \preceq \mu^{\prime}$ which is a contradiction. Hence, $\mu_{l-1} \preceq \mu^{\prime} \prec \mu$.

If $\mu_{l-1}=\mu^{\prime}$, then b) follows from the definition of the iterated sequence of valuations.

If $\mu_{l-1} \prec \mu^{\prime} \prec \mu$, there exists $\phi \in \Phi\left(\mu_{l-1}, \mu^{\prime}\right)=\Phi\left(\mu_{l-1}, \mu\right)=\Phi\left(\mu_{l-1}, \mu_{l}\right)$ such that $\gamma^{\prime}=$ $\mu^{\prime}(\phi)<\mu(\phi)$. (Otherwise, $\mu_{l} \preceq \mu^{\prime}$, which is a contradiction.) Furthermore, $\mu_{\gamma^{\prime}} \preceq \mu^{\prime} \prec \mu$, where $\mu_{\gamma^{\prime}}=\left[\mu_{l-1} ; \mu_{\gamma^{\prime}}(\phi)=\gamma^{\prime}\right]$. If $\mu_{\gamma^{\prime}} \prec \mu^{\prime} \prec \mu$, then $\Phi\left(\mu_{\gamma^{\prime}}, \mu^{\prime}\right)=\Phi\left(\mu_{\gamma^{\prime}}, \mu\right)$ (see Proposition 2.3 (b)) and since $\gamma^{\prime}=\mu_{\gamma^{\prime}}(\phi)=\mu^{\prime}(\phi)<\mu(\phi)$, then $\phi \in \Phi\left(\mu_{\gamma^{\prime}}, \mu^{\prime}\right)$, which is a contradiction. Hence, $\mu_{\gamma^{\prime}}=\mu^{\prime}$ and $\mu_{\gamma^{\prime}}=\mu^{\prime} \prec \mu_{l}$. (This last is clear when $\mu_{l}$ is a limit valuation and follows from Proposition 3.5 (b) when $\mu_{l}$ is an iterated valuation of $\mu_{l-1}$.)

At this point, b) follows easily from the definition of the iterated sequence of valuations.

The next result guarantees the existence of the minimum for the order $\preceq$ of two real valuations.

Proposition 4.2. With the above assumptions and notation, let us consider $\widetilde{\mu} \in \operatorname{Val}(R)$ such that $\mu \npreceq \widetilde{\mu}, \widetilde{\mu} \npreceq \mu$ and assume $\mu^{\prime} \prec \mu$ and $\mu^{\prime} \prec \widetilde{\mu}$ for some $\mu^{\prime} \in \operatorname{Val}(R)$. Then there exists $\mu_{*} \in \operatorname{Val}(R)$ such that $\mu_{*} \prec \mu, \mu_{*} \prec \widetilde{\mu}$ and $\Phi\left(\mu_{*}, \mu\right) \cap \Phi\left(\mu_{*}, \widetilde{\mu}\right)=\emptyset$. In particular, $\left\{\mu^{\prime \prime} \in\right.$ $\left.\operatorname{Val}(R) ; \mu_{*} \prec \mu^{\prime \prime} \preceq \mu\right\} \cap\left\{\mu^{\prime \prime} \in \operatorname{Val}(R) ; \mu_{*} \prec \mu^{\prime \prime} \preceq \widetilde{\mu}\right\}=\emptyset$.

Proof. First, assume $\mu(T)<\widetilde{\mu}(T)$ (similarly if $\widetilde{\mu}(T)<\mu(T)$ ). In this case, $\mu_{0} \prec \widetilde{\mu}, \mu_{0} \prec \mu$ (note that $\mu \npreceq \widetilde{\mu})$ and $d\left(\mu_{0}, \widetilde{\mu}\right)=1$. Furthermore, if $\Phi\left(\mu_{0}, \mu\right) \cap \Phi\left(\mu_{0}, \widetilde{\mu}\right) \neq \emptyset$, then $d\left(\mu_{0}, \mu\right)=1$ and there exists $T+a \in \Phi\left(\mu_{0}, \mu\right) \cap \Phi\left(\mu_{0}, \widetilde{\mu}\right)$ for some $a \in D$. Thus, $\min \{\mu(T), \mu(a)\}=\mu_{0}(T+a)<$ $\min \{\mu(T+a), \widetilde{\mu}(T+a)\}, \mu(T)=\mu(a)=\widetilde{\mu}(a)<\widetilde{\mu}(T)$ and $\widetilde{\mu}(T+a)=\widetilde{\mu}(a)=\mu(a)=\mu_{0}(T+a)$, which is a contradiction. Hence, $\Phi\left(\mu_{0}, \mu\right) \cap \Phi\left(\mu_{0}, \widetilde{\mu}\right)=\emptyset$.

Now, assume $\mu(T)=\widetilde{\mu}(T)$, then $\mu_{0} \prec \widetilde{\mu}$. Since $\mu \npreceq \widetilde{\mu}$, there exists a non-negative integer $1 \leq k<t$ such that $\mu_{k-1} \prec \widetilde{\mu}$ and $\mu_{k} \npreceq \widetilde{\mu}$. We can distinguish two possibilities:

A) $\mu^{\prime \prime} \npreceq \widetilde{\mu}$ for each $\mu^{\prime \prime} \in \operatorname{Val}(R)$ with $\mu_{k-1} \prec \mu^{\prime \prime} \prec \mu_{k}$. In this case, $\Phi\left(\mu_{k-1}, \mu\right) \cap \Phi\left(\mu_{k-1}, \widetilde{\mu}\right)=\emptyset$. (Otherwise, let us consider $\phi \in \Phi\left(\mu_{k-1}, \mu\right) \cap \Phi\left(\mu_{k-1}, \widetilde{\mu}\right)$ and write $\gamma^{\prime \prime}=\min \{\mu(\phi), \widetilde{\mu}(\phi)\}$. Then $\mu_{k-1} \prec \mu_{\gamma^{\prime \prime}} \preceq \mu_{k}$ and $\mu_{\gamma^{\prime \prime}} \preceq \widetilde{\mu}$ which is a contradiction, where $\mu_{\gamma^{\prime \prime}}=\left[\mu_{k-1} ; \mu_{\gamma^{\prime \prime}}(\phi)=\gamma^{\prime \prime}\right]$.)

B) There exists $\mu^{\prime \prime} \in \operatorname{Val}(R)$ with $\mu_{k-1} \prec \mu^{\prime \prime} \prec \mu_{k}$ and $\mu^{\prime \prime} \preceq \widetilde{\mu}$. In this case, for every $\mu^{\prime \prime} \in \operatorname{Val}(R)$ with $\mu_{k-1} \prec \mu^{\prime \prime} \prec \mu_{k}$, there exists $\gamma^{\prime \prime} \in \Lambda\left(\mu_{k-1}, \mu_{k}\right)=\Lambda\left(\mu_{k-1}, \mu\right)$ such that $\mu^{\prime \prime}=\mu_{\gamma^{\prime \prime}}=\left[\mu_{k-1} ; \mu_{\gamma^{\prime \prime}}(\phi)=\gamma^{\prime \prime}\right]$ for some $\phi \in \Phi\left(\mu_{k-1}, \mu\right)$ (see Propositions 3.5 (a) and 3.7).

Let us consider $\gamma_{*}=\sup \left\{\gamma^{\prime \prime} ; \mu_{\gamma^{\prime \prime}} \prec \widetilde{\mu}\right\}$. We point out that $\gamma_{*}<\gamma_{k}=\gamma\left(\mu_{k-1}, \mu\right)$. (Otherwise, $\mu_{k} \preceq \widetilde{\mu}$, which is a contradiction.) Thus, there exists $\phi^{*} \in \Phi\left(\mu_{k-1}, \mu\right)$ such that $\mu\left(\phi^{*}\right)>\gamma_{*}>$ 
$\mu_{k-1}\left(\phi^{*}\right)$ and $\mu_{*}=\mu_{\gamma_{*}}=\left[\mu_{k-1} ; \mu_{\gamma_{*}}(\phi)=\gamma_{*}\right] \prec \widetilde{\mu}$, also by Propositions 3.5 (a) and 3.7. Note that $\mu_{*} \prec \mu_{k}$.

Finally, $\Phi\left(\mu_{*}, \mu\right) \cap \Phi\left(\mu_{*}, \widetilde{\mu}\right)=\emptyset$. Otherwise, there exists $\phi^{\prime} \in \Phi\left(\mu_{*}, \mu\right) \cap \Phi\left(\mu_{*}, \widetilde{\mu}\right)$. Since $\phi^{*} \in$ $\Phi\left(\mu_{*}, \mu\right)$, then $\operatorname{deg}\left(\phi^{*}\right)=\operatorname{deg}\left(\phi^{\prime}\right)=d_{k}$ and $\phi^{\prime} \in \Phi\left(\mu_{k-1}, \mu\right)$. Therefore, $\mu_{*} \prec \mu_{\widehat{\gamma}} \prec \widetilde{\mu}$, where $\widetilde{\gamma}=\min \left\{\mu\left(\phi^{\prime}\right), \widetilde{\mu}\left(\phi^{\prime}\right)\right\}$ and $\mu_{\widetilde{\gamma}}=\left[\mu_{*} ; \mu_{\widetilde{\gamma}}\left(\phi^{\prime}\right)=\widetilde{\gamma}\right]$. Since $\mu_{\widetilde{\gamma}}=\left[\mu_{k-1} ; \mu_{\widetilde{\gamma}}\left(\phi^{\prime}\right)=\widetilde{\gamma}\right]$, then $\mu_{\widehat{\gamma}} \prec \mu_{k}$ which is a contradiction.

As an easy consequence of Lemma 3.10, we have the following result.

Proposition 4.3. With the above assumptions and notation, let us also assume $t \geq 2$, then $d_{i+1} \gamma_{i}<d_{i} \gamma_{i+1}, 1 \leq i<t$.

The relation between the iterated sequence of valuations associated with $\mu$ and the Apéry base of $\mu$ is stated in the following:

Theorem 4.4. With the above assumptions and notation, let $i \geq 1$ be a non negative integer such that $d_{k-1} \leq i<d_{k}$ (here $d_{k}=\infty$ when $k-1=t$ ) and let us write $i=\sum_{j=0}^{k-1} s_{j} d_{j}$ with $0 \leq s_{j}<d_{j+1} / d_{j}, 0 \leq j \leq k-1$. Then $\omega_{i}(\mu)=\sum_{j=0}^{k-1} s_{j} \gamma_{j}$, where $\sum_{j=0}^{k-1} s_{j} \gamma_{j}=\infty$ if $k-1=t$ and $\gamma_{t}=\infty$. In particular, $\omega_{d_{j}}(\mu)=\gamma_{j}, 1 \leq j \leq t$. Moreover, $\omega_{i}(\mu) \leq\left(i / d_{k-1}\right) \gamma_{k-1}$ and the equality holds if and only if $d_{k-1}$ divides $i$.

Proof. The proof is by induction on $k$.

Since $\mu(f)=\mu_{0}(f)$ for each $f \in R$ such that $\operatorname{deg}(f)<d_{1}$, then $\omega_{i}(\mu)=\omega_{i}\left(\mu_{0}\right)=i \mu(T)=i \gamma_{0}$ for $1 \leq i<d_{1}$ and the result is stated for $k=1$.

Let us assume the result true for all non-negative integer $l$ such that $d_{h-1} \leq l<d_{h}$ with $h \leq k-1$. Consider a non-negative integer $i$ with $d_{k-1} \leq i<d_{k}$.

Let $\left\{\beta_{n}^{(h)}\right\}_{n=1}^{\infty} \subset \Lambda\left(\mu_{h-1}, \mu\right)$ be a sequence such that $\lim _{n \rightarrow \infty} \beta_{n}^{(h)}=\gamma_{h}$, for all $1 \leq h \leq k$. (If $\gamma_{h} \in \Lambda\left(\mu_{h-1}, \mu\right)$, we take $\beta_{n}^{(h)}=\gamma_{h}$ for all $n \geq 1$.) We also fix $\phi_{n}^{(h)} \in \Phi\left(\mu_{h-1}, \mu\right)$ such that $\mu\left(\phi_{n}^{(h)}\right)=\mu_{h}\left(\phi_{n}^{(h)}\right)=\beta_{n}^{(h)}, n \geq 1$ and $1 \leq h<k$. (If $\gamma_{h} \in \Lambda\left(\mu_{h-1}, \mu\right)$, we take $\phi_{n}^{(h)}=\phi$ with $\mu(\phi)=\gamma_{h}$ for all $n \geq 1$.) Furthermore, let us write $\mu^{(n)}=\left[\mu_{h-1} ; \mu^{(n)}\left(\phi_{n}^{(h)}\right)=\beta_{n}^{(h)}\right], n \geq 1$.

Let us consider $f \in R$ a monic left skew polynomial with $\operatorname{deg}(f)=i$ and write

$$
f=q_{0}^{(n)}\left(\phi_{n}^{(k-1)}\right)^{s_{k-1}}+q_{1}^{(n)}\left(\phi_{n}^{(k-1)}\right)^{s_{k-1}-1}+\cdots+q_{s_{k-1}}^{(n)}
$$

with $\operatorname{deg}\left(q_{l}^{(n)}\right)<d_{k-1}, 0 \leq l \leq s_{k-1}, n \geq 1$. Then $\mu_{k-1}(f)=\mu(f)=\mu^{(n)}(f) \leq \mu_{k-2}\left(q_{0}^{(n)}\right)+$ $s_{k-1} \beta_{n}^{(k-1)}, n \geq 1$. Since $q_{0}^{(n)}$ is monic, $\operatorname{deg}\left(q_{0}^{(n)}\right)=l=i-s_{k-1} d_{k-1}=\sum_{j=0}^{k-2} s_{j} d_{j}$ and $s_{k-1} \neq 0$, then $\mu_{k-2}\left(q_{0}^{(n)}\right) \leq \omega_{l}(\mu)=\sum_{j=0}^{k-2} s_{j} \gamma_{j}$ by induction hypothesis. Hence, $\mu_{k-1}(f)=\mu(f) \leq$ $\sum_{j=0}^{k-1} s_{j} \gamma_{j}$ and $\omega_{i}(\mu) \leq \sum_{j=0}^{k-1} s_{j} \gamma_{j}$.

On the other hand, if $g_{n}=T^{s_{0}} \prod_{j=1}^{k-1}\left(\phi_{n}^{(j)}\right)^{s_{j}}, n \geq 1$, then $\operatorname{deg}\left(g_{n}\right)=i$ and $\mu\left(g_{n}\right)=\sum_{j=0}^{k-1} s_{j} \beta_{n}^{(j)} \leq$ $\omega_{i}(\mu)$, where $\beta_{n}^{(0)}=\mu(T)=\mu_{0}(T)=\gamma_{0}, n \geq 1$. Therefore, $\lim _{n \rightarrow \infty}\left(\sum_{j=0}^{k-1} s_{j} \beta_{n}^{(j)}\right)=\sum_{j=0}^{k-1} s_{j} \gamma_{j} \leq$ $\omega_{i}(\mu)$. Hence, $\omega_{i}(\mu)=\sum_{j=0}^{k-1} s_{j} \gamma_{j}$.

Finally, since $\gamma_{0} / d_{0}<\gamma_{1} / d_{1}<\cdots<\gamma_{k} / d_{k}$ (see Proposition 4.3), then

$$
\omega_{i}(\mu) \leq s_{k-1} \gamma_{k-1}+\cdots+s_{1} \gamma_{1}+s_{0} \frac{d_{0}}{d_{1}} \gamma_{1}=
$$




$$
\begin{gathered}
=s_{k-1} \gamma_{k-1}+\cdots+s_{2} \gamma_{2}+\frac{s_{1} d_{1}+s_{0} d_{0}}{d_{1}} \gamma_{1} \leq s_{k-1} \gamma_{k-1}+\cdots+s_{2} \gamma_{2}+\frac{s_{1} d_{1}+s_{0} d_{0}}{d_{2}} \gamma_{2}= \\
=s_{k-1} \gamma_{k-1}+\cdots+s_{3} \gamma_{3}+\frac{s_{2} d_{2}+s_{1} d_{1}+s_{0} d_{0}}{d_{2}} \gamma_{2} \leq \cdots \leq \\
\leq s_{k-1} \gamma_{k-1}+\frac{s_{k-2} d_{k-2}+\cdots+s_{1} d_{1}+s_{0} d_{0}}{d_{k-1}} \gamma_{k-1}=\frac{i}{d_{k-1}} \gamma_{k-1} .
\end{gathered}
$$

Note that if $s_{j} \neq 0$ for some $0 \leq j<k-1$, then the corresponding inequality must be strict and this complete the proof.

Notice that Theorem 4.4 shows that the Apéry base $\left\{\omega_{i}(\mu)\right\}_{i \geq 0}$ is determined by the sequence $\left\{\left(d_{i}, \gamma_{i}\right)\right\}_{i \geq 0}$ (i.e. the numerical part of the iterated sequence of valuations associated with $\mu$ ). The converse is also true, that is the Apéry base determines the numerical part of the iterated sequence of valuations. In fact, we have the following:

Corollary 4.5. With the above assumptions and notation, we have

a) $d_{1}=1$ if and only if $\omega_{0}(\mu)<\omega_{1}(\mu)$.

b) For $k>1$ the following statements are equivalent:

$$
\begin{aligned}
& \text { b.1) } \omega_{k}(\mu)>\omega_{r}(\mu)+\omega_{s}(\mu) \text { for each } r \text { and } s \text { with } 1 \leq r, s<k \text { and } r+s=k \text {. } \\
& \text { b.2) } k \in\left\{d_{i} ; d_{i}>1\right\} \text {. }
\end{aligned}
$$

Proof. a) Assume $d_{1}=1$, then $\mu_{0}(T+a)<\mu(T+a)$ for some $a \in D$. If $\mu(a)<\mu(T)$, then $\mu(a)=\mu_{0}(T+a)=\mu(T+a)$ which is a contradiction. Thus, $\mu(a) \geq \mu(T)$ and $\omega_{0}(\mu)=\mu(T) \leq$ $\mu_{0}(T+a)<\mu(T+a) \leq \omega_{1}(\mu)$.

Conversely, assume $\mu(T)=\omega_{0}(\mu)<\omega_{1}(\mu)$, then $\mu(T)<\mu(T+a)$ for some $a \in D$. Thus, $\mu(T)=\mu(a)$ and $\mu(T)=\mu(a)=\mu_{0}(T+a)<\mu(T+a)$. Hence, $d_{1}=1$.

b) To see b.1) $\Longrightarrow$ b.2), assume $k \notin\left\{d_{i} ; d_{i}>1\right\}$, so $d_{i}<k<d_{i+1}$ for some non negative integer $i$ (here $d_{i+1}=\infty$ when $i=t<\infty$ ). We can write $k=\sum_{j=0}^{i} s_{j} d_{j}$ with $0 \leq s_{j}<d_{j+1} / d_{j}$, $0 \leq j \leq i$. We have $\sum_{j=0}^{k-1} s_{j} \geq 2$ (otherwise, $k=d_{j}>1$ ). Now, it follows immediately that $\omega_{k}(\mu)=\omega_{r}(\mu)+\omega_{s}(\mu)$ for some $r$ and $s$ with $1 \leq r, s<k$ and $r+s=k$.

b.2) $\Longrightarrow$ b.1). Assume $k=d_{i}>1$ and let $r$ and $s$ be such that $1 \leq r, s<k$ and $r+s=k$. By Theorem 4.4, $\omega_{r}(\mu) \leq\left(r / d_{i-1}\right) \gamma_{i-1}$ and $\omega_{s}(\mu) \leq\left(s / d_{i-1}\right) \gamma_{i-1}$. Thus, $\omega_{r}(\mu)+\omega_{s}(\mu) \leq$ $\left((r+s) / d_{i-1}\right) \gamma_{i-1}=\left(d_{i} / d_{i-1}\right) \gamma_{i-1}<\gamma_{i}=\omega_{d_{i}}(\mu)$.

\section{The tree structure}

In this section, we fix a proper $\sigma$-compatible valuation $\nu$ on $D$ such that

$$
I(\sigma, \delta, \nu)=\inf \{\nu(\delta(a))-\nu(a) ; a \in D-\{0\}\}>-\infty .
$$

We call $I(\sigma, \delta, \nu)$ the compatibility index of $\nu$ and we write $\operatorname{Val}_{\nu}(R)=\left\{\mu \in \operatorname{Val}(R) ; \mu_{\mid D}=\nu\right\}$. Notice that $\operatorname{Val}_{\nu}(R) \neq \emptyset$, since $I(\sigma, \delta, \nu)>-\infty$. (See Proposition 4.5 of [CZ].)

Next and following [FJ], we recall some definitions about trees.

Let $(\mathcal{T}, \leq)$ be a partially ordered set (poset), we say that a totally ordered subset $S \subset \mathcal{T}$ is full, if $\sigma, \sigma^{\prime} \in S, \tau \in \mathcal{T}$ and $\sigma \leq \tau \leq \sigma^{\prime}$ imply $\tau \in S$.

Definition 5.1. A non-metric tree is a poset $(\mathcal{T}, \leq)$ such that 
(T1) For every $\sigma, \tau \in \mathcal{T}$ there exists $\epsilon \in \mathcal{T}$ with $\epsilon \leq \sigma$ and $\epsilon \leq \tau$;

(T2) for every $\tau \in \mathcal{T}$ the set $\{\sigma \in \mathcal{T} ; \sigma \leq \tau\}$ is order isomorphic to an interval of $\overline{\mathbb{R}}$;

(T3) every full, totally ordered subset of $\mathcal{T}$ is order isomorphic to an interval of $\overline{\mathbb{R}}$.

Furthermore, $\mathcal{T}$ is said complete if every increasing sequence $\left(\tau_{i}\right)_{i \geq 1}$ in $\mathcal{T}$ is bounded above, i.e. there exists $\tau_{\infty} \in \mathcal{T}$ such that $\tau_{i} \leq \tau_{\infty}$ for every $i$.

We point out that the above definition of a non-metric tree does not verifies that every non-empty subset $S$ of $\mathcal{T}$ has an infimum in $\mathcal{T}$. In [N] it is suggested to include this last as another condition to be a non-metric tree (see that paper for more details). In our case, the existence of infimum will be guaranteed by Proposition 4.2.

Definition 5.2. A parameterization of a non-metric tree $(\mathcal{T}, \leq)$ is an increasing (or decreasing) mapping $\alpha: \mathcal{T} \longrightarrow[-\infty,+\infty]$ whose restriction to any full totally ordered subset of $\mathcal{T}$ gives a one to one map onto an interval of $\overline{\mathbb{R}}$.

Our main result is:

Theorem 5.3. With the above assumptions and notation $\left(\operatorname{Val}_{\nu}(R), \preceq\right)$ is a parameterized complete non-metric tree.

First, we define a parameterization on $\left(\operatorname{Val}_{\nu}(R), \preceq\right)$. Namely, $\alpha_{\nu}: \operatorname{Val}_{\nu}(R) \longrightarrow \overline{\mathbb{R}}$ be the map given by $\alpha_{\nu}(\mu)=\gamma_{t} / d_{t}$ when $t \neq \infty$ and $\alpha_{\nu}(\mu)=\lim _{i \rightarrow \infty} \gamma_{i} / d_{i}$ when $t=\infty$. Where $\left\{\left(\mu_{i}, d_{i}, \gamma_{i}\right)\right\}_{i=0}^{t}$ is the iterated sequence of valuations associated with $\mu \in \operatorname{Val}_{\nu}(R)$.

For the proof of Theorem 5.3, we first note that the tree condition (T1), follows from Proposition 4.2. Furthermore, by Proposition $2.3(\mathrm{c}), \operatorname{Val}_{\nu}(R)$ is complete. The rest of the proof is an easy consequence of the following:

Lemma 5.4. Let us consider $\mu^{\prime}, \mu \in \operatorname{Val}_{\nu}(R)$ and denote by $\left\{\left(\mu_{i}, d_{i}, \gamma_{i}\right)\right\}_{i=0}^{t}$ and $\left\{\left(\mu_{i}^{\prime}, d_{i}^{\prime}, \gamma_{i}^{\prime}\right)\right\}_{i=0}^{s}$ the iterated sequence of valuations associated with $\mu$ and $\mu^{\prime}$, respectively. We have the following statements:

(a) $\alpha_{\nu}$ is a strictly increasing map.

(b) $\mathcal{J}_{\mu}=\left\{\mu^{\prime} \in \operatorname{Val}_{\nu}(R) ; \mu^{\prime} \preceq \mu\right\}$ is a full totally ordered subset of $\operatorname{Val}_{\nu}(R)$ for each $\mu \in \operatorname{Val}_{\nu}(R)$ and $\alpha_{\nu}$ is a strictly increasing map whose restriction $\alpha_{\mathcal{J}_{\mu}}$ to $\mathcal{J}_{\mu}$ is an increasing one to one map from $\mathcal{J}_{\mu}$ onto an interval of $\overline{\mathbb{R}}$.

(c) Let $\mathcal{S}$ be a full totally ordered subset of $\operatorname{Val}_{\nu}(R)$, then $\alpha_{\nu}(\mathcal{S})$ is order isomorphic to an interval of $\overline{\mathbb{R}}$ via $\alpha_{\nu}$.

Proof. Assume $\mu^{\prime} \prec \mu$. By Proposition 4.1, $s<\infty$ and we can distinguish two possibilities:

(1) If $\mu^{\prime}(T)<\mu(T), t^{\prime}=0$ and $\mu_{0}^{\prime}=\mu^{\prime} \prec \mu_{0}$. Since $d_{0}^{\prime}=1, \gamma_{0}^{\prime}=\mu^{\prime}(T)$, and $\gamma_{0}=\mu(T)$, we get $\alpha_{\nu}\left(\mu^{\prime}\right)=\frac{\gamma_{0}^{\prime}}{d_{0}^{\prime}}<\frac{\gamma_{0}}{d_{0}} \leq \alpha_{\nu}(\mu)$

(2) If $\mu^{\prime}(T)=\mu(T)$, there exists a non-negative integer $1 \leq l \leq t$ such that $\mu_{l-1} \preceq \mu^{\prime} \prec \mu_{l}$. Moreover, either $\mu_{l-1}=\mu^{\prime}$ and $\alpha_{\nu}\left(\mu^{\prime}\right)=\frac{\gamma_{l-1}}{d_{l-1}}<\frac{\gamma_{t}}{d_{t}}=\alpha_{\nu}(\mu)$, by Proposition 4.3, or $\mu_{l-1} \prec$ $\mu^{\prime} \prec \mu_{l}, \mu^{\prime}=\left[\mu_{l-1}, \gamma_{s}^{\prime}=\mu^{\prime}(\phi)\right]$ and $\alpha_{\nu}\left(\mu^{\prime}\right)=\frac{\gamma_{s}^{\prime}}{d_{s}^{\prime}}<\frac{\gamma_{t}}{d_{t}}=\alpha_{\nu}(\mu)$, where $\phi \in \Phi\left(\mu_{l-1}, \mu^{\prime}\right), s=$ 
$l, \mu_{i}^{\prime}=\mu_{i}, d_{i}^{\prime}=d_{i}, \gamma_{i}^{\prime}=\gamma_{i}$ for $0 \leq i \leq s-1=l-1$ and $d_{s}^{\prime}=d_{t}$. In the last case, note that $\mu_{l-1} \prec \mu^{\prime} \prec \mu$ and we can also apply Proposition 4.3. So, we have proved (a).

By Proposition 4.1 and statement (a) above, to get (b) we must only see that $\alpha_{\mathcal{J}_{\mu}}$ is an onto map.

Let us consider $a$ a real number such that $a<\alpha_{\nu}(\mu)$. There exists a non-negative integer $k$ such that $1 \leq k<t$ and $\gamma_{k-1} / d_{k-1} \leq a<\gamma_{k} / d_{k}$.

If $\gamma_{k-1} / d_{k-1}=a$, then $\alpha_{\nu}\left(\mu_{k-1}\right)=a$.

If $\gamma_{k-1} / d_{k-1}<a<\gamma_{k} / d_{k}$, we can distinguish two possibilities:

(1) $\gamma_{k} \in \Lambda\left(\mu_{k-1}, \mu\right)$. In this case, let $\phi \in \Phi\left(\mu_{k-1}, \mu\right)$ be such that $\mu(\phi)>a d_{k}$ and $\mu^{\prime}=$ $\left[\mu_{k-1} ; \mu^{\prime}(\phi)=d_{k} a\right]$. Then $\mu^{\prime} \preceq \mu$ and $\alpha_{\nu}\left(\mu^{\prime}\right)=a$.

(2) $\gamma_{k} \notin \Lambda\left(\mu_{k-1}, \mu\right)$. In this case, $\mu_{k}$ is the limit valuation associated with the pair $\left(\mu_{k-1}, \mu\right)$. Let $\phi \in \Phi\left(\mu_{k-1}, \mu\right)$ be such that $\mu(\phi)>a d_{k}$. Note that $\mu(\phi)=\gamma<\gamma_{k}$. If $\mu^{\prime}=$ $\left[\mu_{k-1} ; \mu^{\prime}(\phi)=d_{k} a\right]$, then $\mu^{\prime} \preceq \mu$ and $\alpha_{\nu}\left(\mu^{\prime}\right)=a$.

To see (c), we point out that $\mathcal{S}$ is bounded above by Proposition 2.3 (c). Let $\widetilde{\mu} \in \operatorname{Val}_{\nu}(R)$ be an upper bound of $\mathcal{S}$. Thus $\mathcal{S} \subset\left\{\mu \in \operatorname{Val}_{\nu}(R) ; \mu \preceq \widetilde{\mu}\right\}=\mathcal{J}_{\widetilde{\mu}}$ and $\alpha_{\nu}: \mathcal{J}_{\widetilde{\mu}} \rightarrow\left(-\infty, \alpha_{\nu}(\widetilde{\mu})\right]$ is a strictly increasing order isomorphism, by the above statement. Hence, $\alpha_{\nu}(\mathcal{S})$ is a full totally ordered subset of $\left(-\infty, \alpha_{\nu}(\widetilde{\mu})\right]$ and it must be an interval of $\overline{\mathbb{R}}$.

Remark 5.5. Notice that $(\operatorname{Val}(R), \preceq)$ is the disjoint union of $\left(\operatorname{Val}_{\nu}(R), \preceq\right)$ such that $I(\sigma, \delta, \nu)>$ $-\infty$.

On the other hand, it is possible that $I(\sigma, \delta, \nu)=-\infty$ and thus $\operatorname{Val}_{\nu}(R)=\emptyset$. For instance, let $D=K\left(\left\{X_{i}\right\}_{i=1}^{\infty}\right)$ be the quotient field of the polynomial ring $K\left[\left\{X_{i}\right\}_{i=1}^{\infty}\right]$ in a denumerable set of variables $\left\{X_{i}\right\}_{i=1}^{\infty}$ with coefficients in a commutative field $K$. We take $\sigma=1_{D}$ and $\delta$ the $K$-derivation given by $\delta\left(X_{i}\right)=X_{i+1}$. Finally, let $\nu$ be the monomial valuation on $D$ given by $\nu\left(X_{n}\right)=-n^{2}$. Since $\nu\left(\delta\left(X_{n}\right)\right)-\nu\left(X_{n}\right)=-(n+1)^{2}+n^{2}=-(2 n+1)$, we have $I\left(1_{D}, \delta, \nu\right)=-\infty$

\section{Maclane Key Polynomials}

In this section, we shall define left key skew polynomials for Krull valuations in a similar way as in $[M]$. In fact, our concept of left key skew polynomial coincides with MacLane's one when we only consider the polynomial ring in one variable with coefficients in a commutative field, (i.e. when $D$ is a commutative field, $\sigma=1_{D}$ and $\delta=0$ ).

With the notation as in the previous sections, let $\mu \in \operatorname{Val}(R)$ be a Krull real valuation.

Definition 6.1. For any $f, g \in R$ we say:

(1) $f$ is $\mu$-equivalent to $g$, if $\mu(f-g)>\mu(f)=\mu(g)$. We shall denote it by $f \sim_{\mu} g$ or simply by $f \sim g$ when no confusion can arise.

(2) $g$ is left $\mu$-divisible by $f$, if there exists $h \in R$ such that $g \sim_{\mu} h f$.

Definition 6.2. A non-zero element $\phi \in R$ is a left key skew polynomial for $\mu$ if it satisfies the following conditions:

(K.1) Irreducibility. Let $f, g \in R$ be such that $f g$ is left $\mu$-divisible by $\phi$, then one of the factors is left $\mu$-divisible by $\phi$. 
(K.2) Minimal degree. For all $f \in R$ such that $f$ is left $\mu$-divisible by $\phi$, we have $\operatorname{deg}(\phi) \leq$ $\operatorname{deg}(f)$.

(K.3) Monicity. The leading coefficient of $\phi$ is 1.

(K.4) Compatibility. $\mu(\phi)<\min \{\mu(r(\phi, a))-\mu(a) ; a \in R, 0 \leq \operatorname{deg}(a)<\operatorname{deg}(\phi)\}$, where $\phi \cdot a=q(\phi, a) \cdot \phi+r(\phi, a)$, with $\operatorname{deg}(q(\phi, a))=\operatorname{deg}(a)$ and $\operatorname{deg}(r(\phi, a))<\operatorname{deg}(\phi)$.

For a left key skew polynomial $\phi \in R$, we write $I(\sigma, \delta, \mu, \phi)=\min \{\mu(r(\phi, a))-\mu(a) ; a \in R, 0 \leq$ $\operatorname{deg}(a)<\operatorname{deg}(\phi)\}$ and we call $I(\sigma, \delta, \mu, \phi)$ the left compatibility index of $\phi$ with respect to $\mu$. Therefore, the compatibility property means $I(\sigma, \delta, \mu, \phi)>\mu(\phi)$

In a similar way as in Theorem 3.4, we have the following result.

Proposition 6.3. With the above assumptions and notation, let $\phi$ be a left key skew polynomial for $\mu$ and $\tau \in \overline{\mathbb{R}}$ be such that $I(\sigma, \delta, \mu, \phi) \geq \tau>\mu(\phi)$. Let us write $\mu_{\tau}(g)=\min \left\{\mu\left(g_{i}\right)+i \tau ; 0 \leq\right.$ $i \leq r\}$ for each $g \in R$, where $g=\sum_{i=0}^{r} g_{i} \phi^{i}$ with $\operatorname{deg}\left(g_{i}\right)<\operatorname{deg}(\phi), 0 \leq i \leq r$. Then $\mu_{\tau} \in \operatorname{Val}(R)$. Furthermore, $\mu \preceq \mu_{\tau}$ and $\mu_{\tau}(f)=\mu(f)$ for each $f \in R$ such that $\operatorname{deg}(f)<\operatorname{deg}(\phi)$.

We point out that Proposition 6.3 is nothing but Theorem 4.2 of $[\mathrm{M}]$ and as in that paper we call $\mu_{\tau}$ an augmented valuation of $\mu$ and we write $\mu_{\tau}=\left[\mu ; \mu_{\tau}(\phi)=\tau\right]$. (See also section 3.)

The next result characterize left key skew polynomial in terms of the natural partial order on the set $\operatorname{Val}(R)$.

Theorem 6.4. With the above assumptions and notation, let $\phi \in R$ be a monic left skew polynomial. Then $\phi$ is a left key skew polynomial for $\mu$ if and only if there exists $\widetilde{\mu} \in \operatorname{Val}(R)$ such that $\mu \prec \widetilde{\mu}$ and $\phi \in \Phi(\mu, \widetilde{\mu})$.

Proof. The necessary condition is consequence of Proposition 6.3.

For the sufficient condition, since the monicity and compatibility properties with respect to $\mu$ are verified for every $\phi \in \Phi(\mu, \widetilde{\mu})$, we only must prove minimal degree and irreducibility properties with respect to $\mu$.

First, we proof that $\phi$ satisfies the minimal degree property with respect $\mu$. If $f \in R$ is left $\mu$-divisible by $\phi$ and $\operatorname{deg}(f)<\operatorname{deg}(\phi)$, then $\mu(f-h \phi)>\mu(f)=\mu(h \phi)$. Since, $\mu(f)=\widetilde{\mu}(f)$ and $\mu(h \phi)<\widetilde{\mu}(h \phi)$, we get $\mu(f)=\widetilde{\mu}(f)<\min \{\widetilde{\mu}(f-h \phi), \widetilde{\mu}(h \phi)\}$ which is a contradiction.

In order to see the irreducibility property with respect to $\mu$, let $f, g \in R$ be such that $f g$ is left $\mu$-divisible by $\phi$ and assume that neither $f$ nor $g$ are left $\mu$-divisible by $\phi$. Let us consider $h \in R$ such that $\mu(f g-h \phi)>\mu(f g)=\mu(h \phi)$ and write $f=q_{f} \phi+r_{f}$ and $g=q_{g} \phi+r_{g}$ with $0 \leq \operatorname{deg}\left(r_{f}\right), \operatorname{deg}\left(r_{g}\right)<\operatorname{deg}(\phi)$.

Since $f$ is not left $\mu$-divisible by $\phi$, then $\mu\left(r_{f}\right) \leq \mu(f)$. Moreover, if $\mu\left(r_{f}\right)<\mu(f)$, then $\widetilde{\mu}\left(r_{f}\right)=$ $\mu\left(r_{f}\right)<\mu(f) \leq \widetilde{\mu}(f)$ and $\widetilde{\mu}\left(r_{f}\right)=\mu\left(r_{f}\right)=\mu\left(q_{f} \phi\right)<\widetilde{\mu}\left(q_{f} \phi\right)$, which is a contradiction. Hence, $\mu\left(r_{f}\right)=\mu(f)$ and similarly $\mu\left(r_{g}\right)=\mu(g)$.

Furthermore, we have $f g-h \phi=\Delta+r_{f} r_{g}$, where $\Delta=q_{f} \phi q_{g} \phi+r_{f} q_{g} \phi+q_{f} \phi r_{g}-h \phi$. Since $\mu(f g-h \phi)>\mu(f g)=\mu\left(r_{f} r_{g}\right)=\widetilde{\mu}\left(r_{f} r_{g}\right)$, then $\mu(\Delta)=\mu\left(r_{f} r_{g}\right)=\widetilde{\mu}\left(r_{f} r_{g}\right)$. Thus, $\widetilde{\mu}(f g-h \phi) \geq$ $\mu(f g-h \phi)>\widetilde{\mu}\left(r_{f} r_{g}\right)$ and $\widetilde{\mu}(\Delta)>\mu(\Delta)=\widetilde{\mu}\left(r_{f} r_{g}\right)$, which is a contradiction. 
6.1. Examples. To finish the paper, we give two examples for which $I(\sigma, \delta, \mu, \phi)=\infty$. The examples are given under the assumption that $\sigma$ is the identity map on $D$ and the reader can recognize $\phi$ as a central element of $R=D\left[T ; 1_{D}, \delta\right]=D[T ; \delta]$ according with the characterization given by Amitsur Theorem (see [J], Theorem 1.1.32). Note that $I(\sigma, \delta, \mu, \phi)=\infty$ if and only if for every $a \in R$ with $\operatorname{deg}(a)<\operatorname{deg}(\phi)$ there exists $a^{\prime} \in R$ such that $\phi a=a^{\prime} \phi$ if and only if $\phi R \subseteq R \phi$.

6.1.1. Zero characteristic case. Let $D=\mathbb{C}(X ; \sigma)$ be the Ore quotient ring of $\mathbb{C}[X ; \sigma, 0]=\mathbb{C}[X ; \sigma]$, where $\sigma$ is the conjugation automorphism on $\mathbb{C}$. Note that $D$ is a division ring. (See chapter 6 of $[\mathrm{GW}]$ for details on Ore quotient rings.)

Let $\delta$ be the inner derivation on $D$ associated with $i \in \mathbb{C}$ (i.e. $\delta(a)=i a-a i$ for each $a \in D$.) Note that $\delta\left(X^{2 n+1}\right)=2 i X^{2 n+1}$ and $\delta\left(X^{2 n}\right)=0$. We write $R=D\left[T ; 1_{D}, \delta\right]=D[T ; \delta]$.

Let us also write $\operatorname{deg}_{X}$ the usual degree in $\mathbb{C}[X ; \sigma]$ and denote by $\nu$ the valuation $-\operatorname{deg}_{X}$ on $D$. We have $\nu(\delta(P(X))) \geq \nu(P(X))$ for each $P(X) \in \mathbb{C}[X ; \sigma]$. In particular, $\nu(\delta(a)) \geq \nu(a)$ for each $a \in D$. Therefore, we can consider $\mu_{0}: R \longrightarrow \overline{\mathbb{R}}$ the extension of $\nu$ given by $\mu_{0}(T)=0$. (See Proposition 4.5 of $[\mathrm{CZ}]$.)

We note that $T-i$ is a central element of $R$, since $\delta$ is the inner derivation associated with $i$. Moreover, since $T-i$ has degree one, it is easy to check that $T-i$ is a left skew key polynomial for $\mu_{0}$ and obviously $I\left(1_{D}, \delta, \mu_{0}, T-i\right)=\infty$.

6.1.2. Positive characteristic case. Let $D=K(X, Y)$ be the field of rational functions in two variables over a commutative field $K$ of characteristic $p>0$. Let $\nu: D \longrightarrow \overline{\mathbb{R}}$ be the monomial valuation given by $\nu(X)=1$ and $\nu(Y)=\beta$, where $\beta \in \mathbb{R}$ is a non-negative irrational number. Recall that $\nu\left(\sum_{0 \leq i+j \leq h} a_{i j} X^{i} Y^{j}\right)=\min \left\{i+\beta j ; a_{i j} \neq 0\right\}$ for each $\sum_{0 \leq i+j \leq h} a_{i j} X^{i} Y^{j} \in$ $K[X, Y]$.

Let $n$ be a non-negative integer, such that $n-\beta>1 / p, \delta$ the $K$-derivation on $D$ given by $\delta=$ $X^{n} \frac{\partial}{\partial Y}$ and write $R=D\left[T ; 1_{D}, \delta\right]=D[T ; \delta]$. It is easy to check that $\nu(\delta(a))-\nu(a) \geq n-\beta>1 / p$ for each $a \in D$. Let $\mu_{0}: R \longrightarrow \overline{\mathbb{R}}$ be the extension of $\nu$ such that $\mu_{0}(T)=1 / p$. (See Proposition 4.5 of $[\mathrm{CZ}]$.)

Since $\delta^{p}(a)=0$ for each $a \in D$, then $T^{p}-X$ commutes with every element of $D$. Moreover, since $X$ commutes with $T$, then $T^{p}-X$ commutes with every monomial $a T^{j}, a \in D$ and hence with every left skew polynomial of $R$.

Finally, we point out that $T^{p}-X$ is a left key skew polynomial for $\mu_{0}$ with $I\left(\mu_{0}, T^{p}-X\right)=\infty$. Note that we must only see that $T^{p}-X$ verifies irreducibility and minimal degree properties for $\mu_{0}$. This is quite technical and we do not include the proof here.

\section{REFERENCES}

[BR] M. Baker and R. Rumely, Potential Theory and Dynamics on the Berkovich Projective Line. Mathematical Surveys and Monographs 159, (Amer. Math. Soc., Providence RI 2010.)

[Ber] V.G. Berkovich, Spectral Theory and Analytic Geometry over non-Archimedean Fields. Mathematical Surveys and Monographs 33, (Amer. Math. Soc., Providence RI 1990.)

[CZ] R.C. Churchill and Y. Zhang, Irreducibility criteria for skew polynomials. J. Algebra. 322, (2009), $3797-3822$.

[FJ] C. Favre and M. Jonsson, The Valuative Tree. Lec. Notes in Math. 1853. (Springer-Verlag. 2004.)

[Gra1] A. Granja, Apéry basis and polar invariants of plane curve singularities. Pac. J. Math., 140, no. 1, (1989), $85-96$. 
[Gra2] A. Granja, The valuative tree of a two-dimensional regular local ring. Math. Res. Lett., 14, (2004), $19-34$. [GMR] A. Granja, M. C. Martínez and C. Rodríguez, Analytically irreducible polynomials with coefficients in a real-valued field. Proc. Amer. Math. Soc., 138, (2010), 3449-3454.

[GW] K.R. Goodearl and R. B. Warfield, Jr., An Introduction to Noncommutative Noetherian Rings. London Math. Soc. Student Texts 61. (Cambridge University Press. 2004.)

[HOS] F. J. Herrera Govantes, M. A. Olalla Acosta and M. Spivakovsky, Valuations in algebraic field extensions. J. Algebra, 312, (2007), 1033-1074.

[J] N. Jacobson, Finite Dimensional Division Algebras over Fields (Springer-Verlag. 1996.)

[L] H. Li, Valuation extensions of algebras defined by monic Gröbner bases Algebras Representation Theory, (2011), doi:10.1007/s10468-011-9287-1.

[M] S. MacLane, A construction for absolute values in polynomial rings. Trans. Amer. Math. Soc., 40, (1936), $363-395$.

[N] J. Novacoski, Valuations centered at a two-dimensional regular local ring. Infima and Topologies. Preprint.

[V] M. Vaquié, Extension d'une valuation. Trans. Amer. Math. Soc., 359, no 7, (2007), 3439-3481.

Dpto. de Matemáticas, Universidad de León, 24071-León, Spain

E-mail address: angel.granja@unileon.es

Dpto. de Matemática Aplicada, Universidad de Valladolid, 47014-Valladolid, Spain

E-mail address: carmen@mat.uva.es

Dpto. de Matemáticas, Universidad de León, 24071-León, Spain

E-mail address: mcrods@unileon.es 